TRANSACTIONS OF THE

AMERICAN MATHEMATICAL SOCIETY

Volume 350, Number 8, August 1998, Pages 3207-3235

S 0002-9947(98)02305-8

\title{
BASED ALGEBRAS AND STANDARD BASES FOR QUASI-HEREDITARY ALGEBRAS
}

\author{
JIE DU AND HEBING RUI
}

\begin{abstract}
Quasi-hereditary algebras can be viewed as a Lie theory approach to the theory of finite dimensional algebras. Motivated by the existence of certain nice bases for representations of semisimple Lie algebras and algebraic groups, we will construct in this paper nice bases for (split) quasi-hereditary algebras and characterize them using these bases. We first introduce the notion of a standardly based algebra, which is a generalized version of a cellular algebra introduced by Graham and Lehrer, and discuss their representation theory. The main result is that an algebra over a commutative local noetherian ring with finite rank is split quasi-hereditary if and only if it is standardly fullbased. As an application, we will give an elementary proof of the fact that split symmetric algebras are not quasi-hereditary unless they are semisimple. Finally, some relations between standardly based algebras and cellular algebras are also discussed.
\end{abstract}

\section{INTRODUCTION}

Quasi-hereditary algebras were introduced by E. Cline, B. Parshall and L. Scott [S], [CPS1] around 1987 in order to deal with certain categories, called highest weight categories, which arise from the representation theory of semisimple Lie algebras and algebraic groups. Thus, they have important applications to Lie theory. Quasi-hereditary algebras have also been extensively studied in the context of ring theory (see, e.g., [DR1] and [DR2]), where ideas and techniques such as weights, Weyl modules, and good filtrations from the representation theory of Lie algebras are brought into ring theory. So we may regard the introduction of quasi-hereditary algebras as a Lie theory approach to the theory of finite dimensional algebras.

This paper is another attempt to bring some useful ideas and techniques which appeared in the study of Lie algebra representations in the late 70's (see [KL]) into the study of quasi-hereditary algebras. We shall look at these algebras through the existence of certain nice bases which locally determine (or are determined by) bases for standard and costandard modules. The motivation of this work is behind some recent developments on $q$-Schur algebras (see [Du1], [G3], [Gr]) and on cellular algebras [GL]. In [Du1], bases analogous to the Kazhdan-Lusztig bases for Hecke algebras are introduced for $q$-Schur algebras, while in [G3], [Gr] another interesting basis - the $q$-codeterminant basis - is introduced for a $q$-Schur algebra (see also

Received by the editors September 13, 1996.

1991 Mathematics Subject Classification. Primary 16G99, 17B10; Secondary 20C20, 20C30.

Both authors gratefully acknowledge support received from Australian Research Council under Large ARC Grant A69530243. The second author is partially supported by the National Natural Science Foundation, grant no. 19501016, in P.R. China. He wishes to thank the University of New South Wales for its hospitality during his visit. 
[Du2] for a special case). Remarkably, both bases share some properties (see [GL]) such as (a) both respect the partial order on the poset of dominant weights; (b) both give (or are determined by) important bases for standard modules; (c) the quasi-heredity of $q$-Schur algebras can be derived from either of the bases. This observation gives rise to a natural question: Are there such bases for arbitrary quasi-hereditary algebras? We shall answer the question for split quasi-hereditary algebras in this paper.

Motivated from Hecke algebras and their Kazhdan-Lusztig bases (cf. [KL]), we introduce the notion of based algebras $A$ on a poset $\Lambda$ with a defining base $\mathcal{B}$ in section one. These are a class of algebras whose defining bases are fibred over $\Lambda: \mathcal{B}=\bigcup_{\lambda \in \Lambda} \mathcal{B}^{\lambda}$ and the basis elements over a coideal of $\Lambda$ span an ideal of $A$. So we may filter $A$ with a sequence of ideals whose sections, called strata below, are indexed by the elements in $\Lambda$. A Hecke algebra with its KL basis is a based algebra over the poset of two-sided cells. Unlike the situation for Hecke algebras, every single fibre $\mathcal{B}^{\lambda}$ is a two-sided quasi-cell, i.e., a union of two-sided cells in the sense of [Lu, 29.4]. In order to deal with an approximation to one-sided cells, we introduced the notion of standardly based algebras. This is a generalized version of cellular algebras [GL] obtained by dropping the requirement of the existence of a certain involution on $A$. Notice that, by the definition of a standardly based algebra, the fibre $\mathcal{B}^{\lambda}$ of the defining base is only partitioned into left or right quasicells, i.e., unions of left or right cells, and the cardinality of a left quasi-cell may be different from that of right quasi-cells. Bilinear forms can be introduced on each stratum in a standardly based algebra. When the images of these forms are equal to the ground ring, we say that the standardly based algebra is standardly full-based. Such algebras are the main objects investigated in this paper.

In section two, we study the representation theory of standardly based algebras. Standard and costandard modules are defined by the standard defining base. By considering the restriction of the bilinear form to a stratum, we obtain a sufficient condition for the standard modules to be cyclic. When $\mathrm{k}$ is a field, simple modules and their projective covers are discussed. Compare those in [GL].

The main result of the paper is given in sections three and four. After a quick review of integral quasi-hereditary algebras, we prove in section three that every standardly full-based algebra over a commutative noetherian ring $\mathrm{k}$ is quasi-hereditary. If in addition $\mathrm{k}$ is local, then the algebra is split. Thus the defining base gives rise to bases for standard modules in the corresponding highest weight category. In section four, we prove that every split quasi-hereditary algebra $A$ over a commutative local noetherian ring is standardly full-based. The proof of this result gives an inductive construction of a standard defining base via the given bases for every standard (left and right) $A$-module. Thus we establish a correspondence between (global) standard defining bases and (local) bases for standard $A$-modules. So we shall call a standard defining base of $A$ a standard basis. In particular, if $\mathrm{k}$ is an algebraically closed field, then quasi-hereditary algebras over $\mathrm{k}$ can be characterized as standardly full-based algebras. We remark that our proof uses the results on kfinite highest weight categories developed in [DS]. As an application of our theory, we shall present at the end of section four a simple and direct proof for the fact that a split, symmetric and nonsemisimple algebra over a field is not quasi-hereditary.

We shall give two more applications of our main results. In section five, we present two different standard bases for $q$-Schur algebras. The first is obtained by showing an existing basis to be a standard defining base. The second is constructed 
inductively as shown in the proof of Theorem (4.2.1). Standard duality and involutions will be introduced in the last section. We shall prove that every standardly based algebra over a commutative ring with a standard involution is cellular. For split quasi-hereditary algebras, distinguished standard involutions are introduced, and we shall prove that a split quasi-hereditary algebra is cellular if and only if it has a distinguished standard involution.

Our main result also gives a new criterion for integral quasi-hereditary algebras as indicated in the examples (1.3.4) and (5.6.1). In these examples, we give a new proof for the quasi-heredity of Borel subalgebras of Schur and $q$-Schur algebras where the standard defining bases are simply the natural bases. While completing the present paper, the authors learned that a different criterion for those quasihereditary algebras over an integral domain whose extension to the quotient field is semisimple has been obtained by S. König $[\mathrm{K}]$.

\section{BASED AND STANDARDLY BASED ALGEBRAS}

In this section, we shall introduce the notion of based algebras and discuss certain elementary properties of these algebras.

Throughout, let $\mathrm{k}$ be a commutative ring (with 1). By a k-algebra $A$ (or an algebra over $\mathrm{k}$ ) we mean that $A$ is an associative algebra over $\mathrm{k}$ with identity element 1.

1.1 Based algebras. Let $(\Lambda, \leqslant)$ be a partial ordered set. A subset $\Phi \subseteq \Lambda$ is called an ideal of $\Lambda$ if $\lambda \leqslant \mu$ implies $\lambda \in \Phi$ for any $\mu \in \Phi$ and $\lambda \in \Lambda$. A subset $\Gamma$ is said to be a coideal if $\Lambda \backslash \Gamma$ is an ideal.

(1.1.1) Definition. Let $A$ be a k-algebra and $\Lambda$ a poset. $A$ is called a based algebra on $\Lambda$ if the following conditions hold.

(a) $A$ has a basis $\mathcal{B}$, fibred over $\Lambda$. That is, $\mathcal{B}$ is a disjoint union of subsets $\mathcal{B}^{\lambda}$ $(\lambda \in \Lambda)$. The basis $\mathcal{B}$ will be called a defining base.

(b) For any $a \in A$ and $b \in \mathcal{B}^{\lambda}$, the products $a b$ and $b a$ are linear combinations of basis elements $c \in \mathcal{B}^{\mu}$ with $\mu \geqslant \lambda$.

From the definition, one sees easily that, if $\Gamma$ is a coideal of $\Lambda$, then the submodule $A(\Gamma)$ spanned by $\bigcup_{\lambda \in \Gamma} \mathcal{B}^{\lambda}$ is a two-sided ideal of $A$. Thus, if $\Gamma^{\prime}=\Lambda \backslash \Gamma$, then $A\left[\Gamma^{\prime}\right]=A / A(\Gamma)$ is also a based algebra on the poset $\Gamma^{\prime}$.

If $\Lambda$ is finite and ordered linearly,

$$
\Lambda=\left\{\lambda_{1}, \cdots, \lambda_{m}\right\}, \text { compatible with } \leqslant \text {, i.e., } \lambda_{i}>\lambda_{j} \Rightarrow i<j,
$$

then we have a filtration

$$
0=J_{0} \subset J_{1} \subset \cdots \subset J_{m}=A,
$$

where $J_{i}=A\left(\left\{\lambda_{1}, \cdots, \lambda_{i}\right\}\right)$ for all $i=1, \cdots, m$. We shall call (1.1.3) a based filtration of $A$. Note that, for $\lambda \in \Lambda$, the set $\Lambda^{\geqslant \lambda}=\{\mu \in \Lambda \mid \mu \geqslant \lambda\}$ is a coideal of $\Lambda$. Let $A^{\geqslant \lambda}=A\left(\Lambda^{\geqslant \lambda}\right), A^{>\lambda}=A\left(\Lambda^{\geqslant \lambda} \backslash\{\lambda\}\right)$ and $A^{\lambda}=A^{\geqslant \lambda} / A^{>\lambda}$. Thus, $A^{\lambda}$ is an $(A, A)$-bimodule and is also an ideal of the quotient algebra $A / A^{>\lambda}$. Note also that, as a k-module, $A^{\lambda}$ can be regarded as the free submodule of $A$ spanned by $\mathcal{B}^{\lambda}$.

From section two onwards, we shall only consider k-finite based algebras, i.e., $A$ is finitely generated as a $\mathrm{k}$-module. Thus both $\mathcal{B}$ and $\Lambda$ are finite. 
(1.1.4) Examples. (a) Any finite dimensional algebra over a field $\mathrm{k}$ is a based algebra on the ordered set $\{1,2, \cdots, m\}$ if $A$ has a filtration (1.1.3) of two-sided ideals.

(b) Let $\mathcal{H}=\mathcal{H}_{\mathcal{A}, q}$ be the Iwahori-Hecke algebra over $\mathcal{A}=\mathbb{Z}\left[t, t^{-1}\right]\left(q=t^{2}\right)$ associated with a Coxeter system $(W, S)$. Then $\mathcal{H}$ has the Iwahori basis $T_{w}, w \in W$. In [KL] Kazhdan and Lusztig defined a new basis $\mathcal{B}=\left\{B_{w} \mid w \in W\right\}$ for $\mathcal{H}$. $\left(B_{w}\right.$ is denoted by $C_{w}^{\prime}$ in [KL].) Using this basis, $W$ is divided into equivalence classes, called cells, via certain equivalence relations associated to preorders $\leqslant_{L}, \leqslant_{R}, \leqslant_{L R}$. Let $\Lambda$ be the set of two-sided cells with the reversed order. That is, if $\lambda, \mu \in \Lambda$ are two-sided cells of $W$, then $\lambda \leqslant \mu$ iff $x \leqslant L R ~ y$ for any $x \in \mu$ and $y \in \lambda$. By the definition of $\leqslant_{L}$ and $\leqslant_{R}[\mathrm{KL}]$, for any $x, y \in W$ with $x \in \lambda, y \in \mu$ we have

$$
B_{x} B_{y}=\sum_{\substack{z \in \nu \\ \nu \in \Lambda \geqslant \lambda \cap \Lambda \geqslant \mu}} h_{x, y, z} B_{z} .
$$

Therefore, $\mathcal{H}$ is a based algebra on $\Lambda$ with defining base $\mathcal{B}$. Note that $\mathcal{B}^{\lambda}=\left\{B_{w} \mid\right.$ $w \in \lambda\}$ for each $\lambda \in \Lambda$, and a filtration on $\Lambda$ in this case is a cell filtration in the sense that each section of the filtration is a two-sided $\mathcal{H}$-module defined by a two-sided cell.

(c) Let $(W, S)$ be a finite Coxeter group. For any $J \subset S$ let $W_{J}$ be the subgroup of $W$ generated by $J$ and $x_{J}=\sum_{x \in W_{J}} T_{x}$. We define, following [Du4, 1.4],

$$
A=\mathcal{S}_{q}(W)=\operatorname{End}_{\mathcal{H}}\left(\bigoplus_{J \subseteq S} x_{J} \mathcal{H}\right) .
$$

This is a centralising algebra of a $q$-permutation module and has a natural basis indexed by $\mathcal{D}(W)=\bigcup_{I, J \subseteq S} W_{I} \backslash W / W_{J}$. Motivated by the Kazhdan-Lusztig bases for Hecke algebras, the first author introduced [Du4] an analogous basis for $\mathcal{S}_{q}(W)$. Let $\mathcal{B}=\left\{\theta_{D} \mid D \in \mathcal{D}(W)\right\}$ denote this basis. Using this basis, the set $\mathcal{D}(W)$ is divided into two-sided cells and two elements $D, D^{\prime} \in \mathcal{D}(W)$ are in the same two-sided cell if and only if $w_{D}$ and $w_{D^{\prime}}$ are in the same two-sided cell of $W$, where $w_{X}$ denotes the longest word in $X$. Let $\Lambda$ be the set of two-sided cells of $\mathcal{D}(W)$. Clearly, $\Lambda$ can be identified with the set of two-sided cells of $W$. For any $\lambda \in \Lambda$ denote $\mathcal{B}^{\lambda}=\left\{\theta_{D} \mid D \in \mathcal{D}(W), D \in \lambda\right\}$. Then, for any $\theta_{D} \in \mathcal{B}^{\lambda}$ and $\theta_{D^{\prime}} \in \mathcal{B}^{\mu}$, we have

$$
\theta_{D} \cdot \theta_{D^{\prime}} \in \sum_{\theta \in B^{\nu}, \nu \geqslant \lambda, \mu} \mathbb{Z}\left[t, t^{-1}\right] \theta .
$$

Therefore $A$ is a based algebra on $\Lambda$ with the defining base $\mathcal{B}$.

Following [Lu, 29.4], we see that every basis $\mathcal{B}$ for an associative algebra $A$ is divided into cells via certain equivalence relations on $\mathcal{B}$ which are defined as follows:

(1.1.5) Definition. Let $A$ be a $\mathrm{k}$-algebra and $\mathcal{B}$ a k-basis for $A$. Let the elements $c_{b, b^{\prime}, b^{\prime \prime}} \in \mathrm{k}$ with $b, b^{\prime}, b^{\prime \prime} \in \mathcal{B}$ denote the structure constants of $A$, i.e., they satisfy $b b^{\prime}=\sum_{b^{\prime \prime} \in \mathcal{B}} c_{b, b^{\prime}, b^{\prime \prime}} b^{\prime \prime}$. Then for $b, b^{\prime} \in \mathcal{B}$ we say that $b^{\prime} \leqslant_{L} b$ (resp. $\left.b^{\prime} \leqslant_{R} b\right)$ if there are sequences $b_{1}=b, b_{2}, \cdots, b_{n}=b^{\prime}$ and $\beta_{1}, \cdots, \beta_{n-1}$ in $\mathcal{B}$ such that $c_{\beta_{i}, b_{i}, b_{i+1}} \neq 0$ (resp. $c_{b_{i}, \beta_{i}, b_{i+1}} \neq 0$ ) for all $i=1, \cdots, n-1$. These are preorders on $\mathcal{B}$. We define $\leqslant_{L R}$ to be the preorder on $\mathcal{B}$ generated by $\leqslant_{L}$ and $\leqslant_{R}$. For $x \in\{L, R, L R\}$ and $b, b^{\prime} \in \mathcal{B}$ we say $b \sim_{x} b^{\prime}$ if $b \leqslant_{x} b^{\prime} \leqslant_{x} b$. Thus $\sim_{L}, \sim_{R}$ and $\sim_{L R}$ are equivalence 
relations on $\mathcal{B}$. The corresponding equivalence classes are called left, right and two-sided cells of $\mathcal{B}$ respectively.

This definition raises a very general question: How to find the cell decomposition for a given basis. As seen from the case of Iwahori-Hecke algebras, the answer to this question for some "nice" basis is important in the representations of this algebra.

A defining base $\mathcal{B}$ of a based algebra $A$ is a cruder model of those "nice" bases. We know from the definition that $\mathcal{B}$ is partitioned into subsets $\mathcal{B}^{\lambda}$ and, from (1.1.3), we have a two-sided module - a section of (1.1.3) - associated to each subset $\mathcal{B}^{\lambda}$. Furthermore, we have the following.

(1.1.6) Lemma. Let $(A, \mathcal{B})$ be a based algebra on $\Lambda$. Then, for any $\lambda \in \Lambda, \mathcal{B}^{\lambda}$ is a disjoint union of two-sided cells of $\mathcal{B}$.

Proof. By (1.1.1) and (1.1.5), if $b \in \mathcal{B}^{\lambda}$ and $b^{\prime} \in \mathcal{B}^{\mu}$ satisfy $b \leqslant_{L R} b^{\prime}$, then $\lambda \geqslant \mu$. Thus, if $b \sim_{L R} b^{\prime}$ then $\lambda=\mu$.

We shall call $\mathcal{B}^{\lambda}$ a two-sided quasi-cell of $\mathcal{B}$. Clearly, the set of two-sided quasicells is a poset with order induced by $\leqslant_{L R}$ and fibred over $\Lambda$. If $\mathcal{B}^{\lambda}$ is a single two-sided cell for each $\lambda \in \Lambda$, then (1.1.3) becomes a cell filtration, as seen in the example (1.1.4).

1.2 Standardly based algebras. The based algebras tell us nothing about left or right cells. In order to create a model for those one-sided cells we introduce the following.

(1.2.1) Definition. Let $A$ be a based algebra on the poset $\Lambda$ with defining base $\mathcal{B}=\bigcup_{\lambda \in \Lambda} \mathcal{B}^{\lambda}$. We say that the defining base $\mathcal{B}$ is standard if the following conditions hold:

(a) For any $\lambda \in \Lambda$ there are index sets $I(\lambda)$ and $J(\lambda)$ such that

$$
\mathcal{B}^{\lambda}=\left\{a_{i j}^{\lambda} \mid(i, j) \in I(\lambda) \times J(\lambda)\right\}
$$

(b) For any $a \in A, a_{i j}^{\lambda} \in \mathcal{B}$, we have

$$
\begin{aligned}
& a \cdot a_{i j}^{\lambda} \equiv \sum_{i^{\prime} \in I(\lambda)} f_{i^{\prime}, \lambda}(a, i) a_{i^{\prime} j}^{\lambda} \bmod \left(A^{>\lambda}\right), \\
& a_{i j}^{\lambda} \cdot a \equiv \sum_{j^{\prime} \in J(\lambda)} f_{\lambda, j^{\prime}}(j, a) a_{i j^{\prime}}^{\lambda} \bmod \left(A^{>\lambda}\right),
\end{aligned}
$$

where $f_{i^{\prime}, \lambda}(a, i), f_{\lambda, j^{\prime}}(j, a) \in \mathrm{k}$ are independent of $j$ and $i$, respectively.

A based algebra $(A, \mathcal{B})$ is said to be standardly based if $\mathcal{B}$ is standard. We shall also call $\mathcal{B}$ a defining base of the standardly based algebra $A$.

We observe from (1.2.2) that the k-span of the elements $\left\{a_{i j}^{\lambda}\right\}$, where $j$ is constant, defines a submodule of the $\lambda$-quotient $A^{\lambda}$ for the left action of $A$ by multiplication, and the $\mathrm{k}$-span of the elements $\left\{a_{i j}^{\lambda}\right\}$ with $i$ constant defines a submodule of $A^{\lambda}$ for the right action of $A$ by multiplication. These submodules form a direct sum decomposition of $A^{\lambda}$ both as a left $A$-module and as a right $A$-module, and the summands in each case are all isomorphic. This will be discussed further in section two.

Clearly, if $\alpha$ is an automorphism of a standardly based algebra $A$, then $\alpha(\mathcal{B})$ is another defining base for $A$. Let $A^{\text {op }}$ denote the opposite algebra of $A$. If $A$ is standardly based with defining base $\mathcal{B}=\left\{a_{i j}^{\lambda}\right\}$, then so is $A^{\text {op }}$ with defining base 
$\mathcal{B}^{\text {op }}=\left\{b_{j i}^{\lambda}\right\}$ where $b_{j i}^{\lambda}=a_{i j}^{\lambda}$. Likewise, if $\alpha$ is an anti-automorphism of $A$, then $\alpha(\mathcal{B})$ is another defining base for $A^{\text {op }}$.

Two standard defining bases $\mathcal{B}$ and $\mathcal{B}^{\prime}$ are said to be equivalent if, for all $a \in A$ and all pairs $(i, j) \in I(\lambda) \times J(\lambda)$, the constants $f_{i^{\prime}, \lambda}(a, i)$ and $f_{\lambda, j^{\prime}}(j, a)$ with respect to $\mathcal{B}$ are equal to their counterparts with respect to $\mathcal{B}^{\prime}$.

From (1.2.2) we may introduce, for each $\lambda \in \Lambda$, a function $f_{\lambda}: J(\lambda) \times I(\lambda) \rightarrow \mathrm{k}$ defined as follows: For any $a_{i j}^{\lambda}, a_{i^{\prime} j^{\prime}}^{\lambda} \in \mathcal{B}^{\lambda}$, there is a unique element $f_{\lambda}\left(j, i^{\prime}\right) \in \mathrm{k}$ such that

$$
a_{i j}^{\lambda} a_{i^{\prime} j^{\prime}}^{\lambda} \equiv f_{\lambda}\left(j, i^{\prime}\right) a_{i j^{\prime}}^{\lambda} \bmod \left(A^{>\lambda}\right) .
$$

Note that standardly based algebras are a generalized version of cellular algebras, introduced by Graham and Lehrer [GL]. Thus, every cellular algebra is standardly based. In this generalization, we require neither that $A$ has an involutive antiautomorphism, nor that $I(\lambda)$ is equal to $J(\lambda)$. The significance of this generalization will be shown in section four, where we shall prove that split quasi-hereditary algebras are standardly based algebras. However, if a particular involutive antiautomorphism is in force, then one can easily see the following.

(1.2.4) Lemma. Let $A$ be a standardly based algebra on $\Lambda$ with defining base

$$
\mathcal{B}=\left\{a_{i j}^{\lambda} \mid \lambda \in \Lambda,(i, j) \in I(\lambda) \times J(\lambda)\right\} .
$$

If there is an involutive anti-automorphism $\iota: A \rightarrow A$ such that $\left(a_{i j}^{\lambda}\right)^{\iota}=a_{j i}^{\lambda}$, then $A$ is a cellular algebra.

A further discussion on the relationship with cellular algebras will be given in section six.

For standardly based algebras, we do know something about one-sided cells of the defining base immediately from the definition. Compare (1.1.6).

(1.2.5) Lemma. Let $(A, \mathcal{B})$ be a standardly based algebra on $\Lambda$. Then, for any $a_{i j}^{\lambda}, a_{i^{\prime} j^{\prime}}^{\mu} \in \mathcal{B}$, we have the following.

(a) If $a_{i j}^{\lambda} \leqslant{ }_{L} a_{i^{\prime} j^{\prime}}^{\mu}$ then $\lambda \geqslant \mu$. Thus, $a_{i j}^{\lambda} \sim_{L} a_{i^{\prime} j^{\prime}}^{\mu}$ implies $\lambda=\mu$ and $j=j^{\prime}$.

(b) If $a_{i j}^{\lambda} \leqslant_{R} a_{i^{\prime} j^{\prime}}^{\mu}$ then $\lambda \geqslant \mu$. Thus, $a_{i j}^{\lambda} \sim_{R} a_{i^{\prime} j^{\prime}}^{\mu}$ implies $\lambda=\mu$ and $i=i^{\prime}$.

So, for any $\lambda \in \Lambda$ and $(i, j) \in I(\lambda) \times J(\lambda)$, if we put

$$
\mathcal{B}_{I(\lambda), j}=\left\{a_{i^{\prime} j}^{\lambda} \in \mathcal{B} \mid i^{\prime} \in I(\lambda)\right\} \text { and } \mathcal{B}_{i, J(\lambda)}=\left\{a_{i j^{\prime}}^{\lambda} \in \mathcal{B} \mid j^{\prime} \in J(\lambda)\right\},
$$

then both $\mathcal{B}_{I(\lambda), j}$ and $\mathcal{B}_{i, J(\lambda)}$ are disjoint unions of left and right cells, called left and right quasi-cells respectively.

A standardly based algebra $A$ is said to be crystallographic if $a_{i j}^{\lambda} \leqslant_{X} a_{k l}^{\lambda}(X=$ $L, R)$ implies $a_{i j}^{\lambda} \sim_{X} a_{k l}^{\lambda}$.

Clearly, for a standardly based algebra $A$ satisfying $f_{\lambda} \neq 0$ for all $\lambda \in \Lambda$ and the crystallographic condition, both $\mathcal{B}_{I(\lambda), j}$ and $\mathcal{B}_{i, J(\lambda)}$ are single left and right cells of $\mathcal{B}$ respectively, and therefore every $\mathcal{B}^{\lambda}$ is a single two-sided cell. Thus, the decomposition into cells of a defining base for $A$ can be easily described as follows.

$$
\begin{array}{lll}
\text { Two-sided cell : } & \mathcal{B}^{\lambda} & (\lambda \in \Lambda), \\
\text { Left cells : } & \mathcal{B}_{I(\lambda), j} & (\lambda \in \Lambda, j \in J(\lambda)), \\
\text { Right cells : } & \mathcal{B}_{i, J(\lambda)} & (\lambda \in \Lambda, i \in I(\lambda)) .
\end{array}
$$


Recall that, as a k-module, $A^{\lambda}$ has a basis $\mathcal{B}^{\lambda}$. Let $\beta_{\lambda}\left(a_{i j}^{\lambda}, a_{i^{\prime} j^{\prime}}^{\lambda}\right)=f_{\lambda}\left(j^{\prime}, i\right)$ and extend it bilinearly to $A^{\lambda} \times A^{\lambda}$. Then we obtain a bilinear function $\beta_{\lambda}$ on $A^{\lambda} \times A^{\lambda}$ which is associative as shown below.

(1.2.6) Lemma. For any $x, y \in A^{\lambda}$ and $a \in A$, we have

$$
\beta_{\lambda}(a x, y)=\beta_{\lambda}(x, y a) \text {. }
$$

Proof. By bilinearity, it suffices to prove the case when $x=a_{i j_{0}}^{\lambda}, y=a_{i_{0} j}^{\lambda}$. Using (1.2.2), we have

$$
\begin{aligned}
\beta_{\lambda}\left(a_{i j_{0}}^{\lambda}, a_{i_{0} j}^{\lambda} a\right) & =\sum_{j^{\prime}} f_{\lambda, j^{\prime}}(j, a) \beta_{\lambda}\left(a_{i j_{0}}^{\lambda}, a_{i_{0} j^{\prime}}^{\lambda}\right) \\
& =\sum_{j^{\prime}} f_{\lambda, j^{\prime}}(j, a) f_{\lambda}\left(j^{\prime}, i\right) .
\end{aligned}
$$

and

$$
\begin{aligned}
\beta_{\lambda}\left(a a_{i j_{0}}^{\lambda}, a_{i_{0} j}^{\lambda}\right) & =\sum_{i^{\prime}} f_{i^{\prime}, \lambda}(a, i) \beta_{\lambda}\left(a_{i^{\prime} j_{0}}^{\lambda}, a_{i_{0} j}^{\lambda}\right) \\
& =\sum_{i^{\prime}} f_{i^{\prime}, \lambda}(a, i) f_{\lambda}\left(j, i^{\prime}\right) .
\end{aligned}
$$

By the associativity, we have

$$
\begin{aligned}
\beta_{\lambda}\left(a a_{i j_{0}}^{\lambda}, a_{i_{0} j}^{\lambda}\right) a_{i_{0} j_{0}}^{\lambda} & \equiv a_{i_{0} j}^{\lambda}\left(a \cdot a_{i j_{0}}^{\lambda}\right) \\
& \equiv\left(a_{i_{0} j}^{\lambda} a\right) a_{i j_{0}}^{\lambda} \equiv \beta_{\lambda}\left(a_{i j_{0}}^{\lambda}, a_{i_{0} j}^{\lambda} a\right) a_{i_{0} j_{0}}^{\lambda} \quad \bmod \left(A^{>\lambda}\right),
\end{aligned}
$$

forcing the coefficients to be equal.

(1.2.7) Remark. There is another bilinear form on $A^{\lambda}$ defined by setting

$$
\gamma_{\lambda}\left(a_{i j}^{\lambda}, a_{i^{\prime} j^{\prime}}^{\lambda}\right)=f_{\lambda}\left(j, i^{\prime}\right) .
$$

In this case the associativity property should be replaced by

$$
\gamma_{\lambda}(x a, y)=\gamma_{\lambda}(x, a y) .
$$

Clearly, we have $\beta_{\lambda}(x, y)=\gamma_{\lambda}(y, x)$, and hence $\operatorname{im}\left(\beta_{\lambda}\right)=\operatorname{im}\left(\gamma_{\lambda}\right)$ for all $\lambda \in \Lambda$. We shall use $\beta_{\lambda}$ (not $\gamma_{\lambda}$ ) to define certain useful bilinear functions in 2.3 which in the case of cellular algebras agree with the ones discussed in [GL, §2].

1.3 Standardly full-based algebras. For each $\lambda \in \Lambda$, one sees clearly that the image $\operatorname{im}\left(\beta_{\lambda}\right)$ is an ideal of $\mathrm{k}$. This leads to the following definition.

(1.3.1) Definition. A standardly based algebra $(A, \mathcal{B})$ on $\Lambda$ is said to be standardly full-based if, for each $\lambda \in \Lambda, \operatorname{im}\left(\beta_{\lambda}\right)=\mathrm{k}$.

The adjective full used here will be justified again in (2.4.2) by showing that the set of simple modules of such an algebra $A$ over a field is indexed by the full set $\Lambda$. However, the fullness here of these images reflects also some structure of the algebra.

For a prime ideal $\mathfrak{p}$ of $k$, the localization $k_{\mathfrak{p}}$ of $\mathrm{k}$ at $\mathfrak{p}$ is a local ring. Let $\mathrm{k}(\mathfrak{p})$ be its residue field. For any $\mathrm{k}$-module $M$, let $M(\mathfrak{p})=M \otimes_{\mathrm{k}} \mathrm{k}(\mathfrak{p})$. If $J$ is an ideal of $A$, define $\bar{J}(\mathfrak{p})$ to be the image of $J(\mathfrak{p})$ in $A(\mathfrak{p})$.

(1.3.2) Lemma. Keep the notation introduced above. Let $A$ be a standardly based algebra on $\Lambda$. Then $A$ is standardly full-based if and only if, for each $\lambda \in \Lambda$, the ideal $A^{\lambda}$ of $A / A^{>\lambda}$ is idempotent. 
Proof. Let $J=A^{\lambda}$. The result is clearly true if $\mathrm{k}$ is a field, since

$$
J^{2}=J \Leftrightarrow f_{\lambda}(j, i) \neq 0 \text { for some } i \in I(\lambda), j \in J(\lambda) \Leftrightarrow \operatorname{im}\left(\beta_{\lambda}\right)=\mathrm{k} .
$$

In general, if $\operatorname{im}\left(\beta_{\lambda}\right)=\mathrm{k}$ for all $\lambda$, then the $\mathrm{k}(\mathfrak{p})$-algebra $A(\mathfrak{p})$ is standardly fullbased. Therefore, $\bar{J}(\mathfrak{p})^{2}=\bar{J}(\mathfrak{p})$. So, by [CPS3, 3.3.1], we obtain $\left(J / J^{2}\right)_{\mathfrak{p}}=0$ for all prime ideals $\mathfrak{p}$. Therefore, $J^{2}=J$. Conversely, suppose $J^{2}=J$ but $\operatorname{im}\left(\beta_{\lambda}\right) \neq \mathrm{k}$. Then $0=J(\mathfrak{m})^{2} \neq J(\mathfrak{m})$, for any maximal ideal $\mathfrak{m}$ of $\mathrm{k}$ with $\operatorname{im}\left(\beta_{\lambda}\right) \subseteq \mathfrak{m} \subset \mathrm{k}$, a contradiction.

We shall see in section three that standardly full-based algebras are quasihereditary. The following gives examples of standardly (full-)based algebras.

(1.3.3) Example. A Schur algebra and its Borel subalgebras are standardly based algebras. For Schur algebras, the poset is the set $\Lambda^{+}(n, r)$ of partitions of $r$ with at most $n$ parts, and a defining base is the codeterminant bases defined in [G3, $\S 6]$. For each $\lambda \in \Lambda^{+}(n, r), I(\lambda)=J(\lambda)$ is the set of all semi-standard $\lambda$-tableaux. We leave the details in this case to the reader (see the case of $q$-Schur algebras in section five). We now look at the Borel subalgebras. For any positive integers $n$ and $r$, let

$$
I=I(n, r)=\left\{\left(i_{1}, \cdots, i_{r}\right) \mid 1 \leqslant i_{j} \leqslant n\right\},
$$

and let the symmetric group $\mathfrak{S}_{r}$ act on $I(n, r)$ by place permutation. Thus the centralizing algebra of the corresponding permutation module over $\mathrm{k}$ is called a Schur algebra, denoted $S_{\mathrm{k}}(n, r)$. It has a natural basis $\xi_{\mathcal{O}}$ indexed by the $\mathfrak{S}_{r}$-orbits $\mathcal{O}$ of $I \times I$. Let $\Omega$ be a transversal of the set of all $\mathfrak{S}_{r}$-orbits of $I \times I$ and write, for $(i, j) \in I \times I, \xi_{i j}=\xi_{\mathcal{O}}$ if $(i, j) \in \mathcal{O}$.

Following J.A. Green, we order $\Omega$ by setting $i \preccurlyeq j$ if and only if $i_{1} \leqslant j_{1}, \ldots, i_{r} \leqslant$ $j_{r}$, and we let $S^{\preccurlyeq}$ (resp. $S^{\succcurlyeq}$ ) be the subalgebra spanned by $\xi_{i j}$ with $(i, j) \in \Omega^{\preccurlyeq}$ (resp. $(i, j) \in \Omega^{\succcurlyeq}$ ), where

$$
\left.\Omega^{\preccurlyeq}=\{(i, j) \in \Omega \mid i \preccurlyeq j\} \quad \text { (resp. } \Omega^{\succcurlyeq}=\{(i, j) \in \Omega \mid i \succcurlyeq j\}\right) .
$$

These are called Borel subalgebras. We also introduce the subalgebras $S^{\prec}$ (resp. $S^{\succ}$ ) with identity by using the subset $\Omega_{1}^{\prec}$ (resp. $\Omega_{1}^{\succ}$ ) defined in an obvious way. The subscript 1 here indicates that both sets include 1 which corresponds to the identity element of $S^{\prec}$ or $S^{\succ}$, respectively.

Let $\Lambda(n, r)$ be the set of all compositions $\lambda=\left(\lambda_{1}, \cdots, \lambda_{n}\right)$ of $r$ with $n$ parts. Then $\Lambda(n, r)$ can be identified with the $\mathfrak{S}_{r}$-orbits of $I$. We say that $i \in I$ has weight $\lambda=\operatorname{wt}(i) \in \Lambda(n, r)$ if $i$ is in the corresponding orbit. $\Lambda(n, r)$ has a partial order defined by setting $\lambda \leqslant \mu$ if and only if $\sum_{i=1}^{k} \lambda_{i} \leqslant \sum_{i=1}^{k} \mu_{i}$ for all $k$ with $1 \leqslant k \leqslant n$. Clearly, if $i, j \in I$ have weights $\lambda, \mu$ respectively, then $i \preccurlyeq j \Rightarrow \lambda \geqslant \mu$.

Now we have the following.

(1.3.4) Proposition. The k-algebra $S^{\preccurlyeq}$ (resp. $S^{\succ}, S^{\prec}, S^{\succ}$ ) is a standardly based algebra with defining base over $\Omega^{\preccurlyeq}$ (resp. $\Omega^{\succcurlyeq}, \Omega_{1}^{\prec}, \Omega_{1}^{\succ}$ ). Moreover, $S^{\preccurlyeq}$ and $S^{\succcurlyeq}$ are standardly full-based algebras.

Proof. For any $(i, j) \in \Omega^{\preccurlyeq}$, if $\lambda$ is the weight of $i$, we let $a_{i, j}^{\lambda}=\xi_{i, j}$ and put, for a fixed $(i, j) \in \Omega^{\preccurlyeq}$ with $\lambda$ being the weight of $i$,

$$
I(\lambda)=\{i\} \text { and } J(\lambda)=\left\{j \in I \mid(i, j) \in \Omega^{\preccurlyeq}\right\} .
$$


Thus, for any $(i, j) \in I(\lambda) \times J(\lambda)$ and $\left(i^{\prime}, j^{\prime}\right) \in I(\mu) \times J(\mu)$, we have $a_{i^{\prime} j^{\prime}}^{\mu} a_{i j}^{\lambda}=0$ if $\operatorname{wt}\left(j^{\prime}\right) \neq \operatorname{wt}(i)=\lambda$, and

$$
a_{i^{\prime} j^{\prime}}^{\mu} a_{i j}^{\lambda}=\sum_{\left(i^{\prime} j^{\prime \prime}\right) \in \Omega^{\preccurlyeq}} c_{j^{\prime \prime}} a_{i^{\prime} j^{\prime \prime}}^{\mu}
$$

if $\operatorname{wt}\left(j^{\prime}\right)=\operatorname{wt}(i)=\lambda$, by the product formula in [G2, 2.6-2.7]. Clearly, (1.2.2) holds. This shows that $S^{\preccurlyeq}$ is a standardly based algebra on the poset $\Lambda(n, r)$ with defining base $\left\{\xi_{i j} \mid(i, j) \in \Omega^{\preccurlyeq}\right\}$. Moreover, $\beta_{\lambda}\left(a_{i j}^{\lambda}, a_{i i}^{\lambda}\right)=1$ since $a_{i i}^{\lambda} a_{i j}^{\lambda}=a_{i j}^{\lambda}$. Therefore, $S^{\preccurlyeq}$ is a standardly full-based algebra.

Let $\Lambda(n, r)_{0}=\Lambda(n, r) \cup\{0\}$ and extend the partial order of $\Lambda(n, r)$ to $\Lambda(n, r)_{0}$ by setting $\lambda>0$. Let $I(0)=J(0)=\{0\}$ and $a_{00}^{0}=1$, and define $I(\lambda), J(\lambda)$ and $a_{i j}^{\lambda}$ similarly as above. Now, one sees easily that $S^{\prec}$ is a standardly based algebra on the poset $\Lambda(n, r)_{0}$ with defining base $\Omega_{1}^{\prec}$. In this case, we have $\beta_{\lambda}=0$ for all $\lambda \in \Lambda(n, r)$ and $\beta_{0} \equiv 1$. Therefore, it is not full.

The proof for $S^{\succ}$ and $S^{\succ}$ is almost the same.

We shall see in section three that we have proved that $S^{\preccurlyeq}$ and $S^{\succcurlyeq}$ are quasihereditary over $\mathrm{k}$. This fact when $\mathrm{k}$ is a field was established by Green (see [G2]). The proof here is new.

\section{REPRESEnTATIONS OF STANDARDLY BASED ALGEBRAS}

In this section we assume that $A$ is a standardly based algebra on $\Lambda$ with defining base $\mathcal{B}$. We also assume that $\mathcal{B}$ is finite. Let $A^{\text {op }}$ denote the opposite algebra of $A$. We denote by $A$-mod $(\operatorname{resp}$. $\bmod -A)$ the category of all finitely generated left (resp. right) $A$-modules. So we may identify $\bmod -A$ with $A^{\mathrm{op}}$-mod. Note that the $\mathrm{k}$-linear dual is a functor $(-)^{*}=\operatorname{Hom}_{\mathrm{k}}(-, \mathrm{k}): \bmod -A \rightarrow A$-mod. Throughout, the term module means left module.

If $\Theta$ is a class of (left, right or bi) $A$-modules (closed under isomorphism), we shall use $\mathcal{F}(\Theta)$ to denote the class of all $A$-modules $M$ which have a $\Theta$-filtration, that is, a filtration $0=M_{0} \subseteq M_{1} \subseteq \cdots \subseteq M_{m}=M$ such that all sections $M_{t} / M_{t-1}$, $1 \leqslant t \leqslant m$, belong to $\Theta$.

2.1 Standard and costandard modules. Recall, for any $\lambda \in \Lambda$, that both $A^{\geqslant \lambda}$ and $A^{>\lambda}$ are ideals of $A$. So their quotient $A^{\lambda}=A^{\geqslant \lambda} / A^{>\lambda}$ is a $\mathrm{k}$-free $(A, A)$ bimodule with basis $\mathcal{B}^{\lambda}$ (by abuse of notation). We may also view $A^{\lambda}$ as a ksubmodule of $A$ spanned by $\mathcal{B}^{\lambda}$.

(2.1.1) Lemma. (a) For each $\lambda \in \Lambda$ and $(i, j) \in I(\lambda) \times J(\lambda)$, let $\Delta(\lambda, j)$ (resp. $\Delta(i, \lambda)$ ) be the left (resp. right) A-submodule of $A^{\lambda}$ generated by (the image of) $\mathcal{B}_{I(\lambda), j}\left(\right.$ resp. $\left.\mathcal{B}_{i, J(\lambda)}\right)$. Then $\Delta(\lambda, j)$ (resp. $\left.\Delta(i, \lambda)\right)$ is $\mathrm{k}$-free with basis (the image of) $\mathcal{B}_{I(\lambda), j}$ (resp. $\mathcal{B}_{i, J(\lambda)}$.

(b) $\Delta(\lambda, j)$ (resp. $\Delta(i, \lambda)$ ) is isomorphic to $\Delta\left(\lambda, j^{\prime}\right)$ (resp. $\Delta\left(i^{\prime}, \lambda\right)$ ) for any $\left(i^{\prime}, j^{\prime}\right) \in I(\lambda) \times J(\lambda)$.

Proof. By symmetry, it suffices to consider the case of left $A$-modules.

(a) By (1.2.2), one sees easily that $\Delta(\lambda, j)$ is generated as a k-module by $\mathcal{B}_{I(\lambda), j}$. The freeness is obvious.

(b) By (a) we first have a k-module isomorphism $f: \Delta(\lambda, j) \rightarrow \Delta\left(\lambda, j^{\prime}\right)$ sending $a_{x j}^{\lambda}$ to $a_{x, j^{\prime}}^{\lambda}$ for any $x \in I(\lambda)$. By (1.2.2) again, we see that $f$ is $A$-linear since $f_{i^{\prime}, \lambda}(a, i)$ is independent of $j$. 
(2.1.2) Definition. For $\lambda \in \Lambda$, we define $\Delta(\lambda)$ (resp. $\Delta^{\mathrm{op}}(\lambda)$ ) to be the left (resp. right) $A$-module with k-basis $\left\{a_{i}^{\lambda} \mid i \in I(\lambda)\right\}$ (resp. $\left\{b_{j}^{\lambda} \mid j \in J(\lambda)\right\}$ ) and module action defined by

$$
a a_{i}^{\lambda}=\sum_{i^{\prime} \in I(\lambda)} f_{i^{\prime}, \lambda}(a, i) a_{i^{\prime}}^{\lambda}
$$

$$
\text { (resp. } \left.b_{j}^{\lambda} a=\sum_{j^{\prime} \in J(\lambda)} f_{\lambda, j^{\prime}}(j, a) b_{j^{\prime}}^{\lambda}\right) .
$$

Let $\nabla(\lambda)=\Delta^{\mathrm{op}}(\lambda)^{*}$. We shall call the modules $\Delta(\lambda)$ and $\nabla(\lambda)$ the standard and costandard modules of $A$ (or of the category $A$-mod), respectively.

Clearly, we have

$$
\begin{aligned}
& \Delta(\lambda) \cong \Delta\left(\lambda, j_{0}\right) \text { via } a_{i}^{\lambda} \mapsto a_{i j_{0}}^{\lambda}, \\
& \Delta^{\mathrm{op}}(\lambda) \cong \Delta\left(i_{0}, \lambda\right) \text { via } b_{j}^{\lambda} \mapsto a_{i_{0} j}^{\lambda},
\end{aligned}
$$

for all $i_{0} \in I(\lambda)$ and $j_{0} \in J(\lambda)$.

(2.1.5) Lemma. For any $\lambda \in \Lambda$, the tensor product $\Delta(\lambda) \otimes_{\mathrm{k}} \Delta^{\mathrm{op}}(\lambda)$ is an $(A, A)$ bimodule in a natural way and is isomorphic to $A^{\lambda}$ as bimodule. In particular, as left $A$-module, $A^{\lambda}$ is isomorphic to the direct sum, $\Delta(\lambda)^{\oplus r_{\lambda}}$, of $r_{\lambda}=|J(\lambda)|$ copies of $\Delta(\lambda)$.

Proof. Since $\Delta(\lambda) \otimes_{\mathrm{k}} \Delta^{\mathrm{op}}(\lambda)$ is $\mathrm{k}$-free with basis $a_{i}^{\lambda} \otimes b_{j}^{\lambda},(i, j) \in I(\lambda) \times J(\lambda)$, we have a $\mathrm{k}$-linear isomorphism

$$
m: \Delta(\lambda) \otimes_{\mathrm{k}} \Delta^{\mathrm{op}}(\lambda) \rightarrow A^{\lambda} ; a_{i}^{\lambda} \otimes b_{j}^{\lambda} \rightarrow a_{i j}^{\lambda} .
$$

Now (1.2.2) and (2.1.3) show that this is actually a bimodule isomorphism.

Note that the map $m$ is not the map induced by the multiplication map from $\Delta\left(\lambda, j_{0}\right) \otimes_{\mathrm{k}} \Delta\left(i_{0}, \lambda\right)$ to $A^{\lambda}$. However, if there exist $i_{0}, j_{0}$ such that $f_{\lambda}\left(j_{0}, i_{0}\right)$ is a unit in $\mathrm{k}$, then $m$ can be defined by the multiplication map

$$
\text { mult : } \Delta\left(\lambda, j_{0}\right) \otimes_{\mathrm{k}} \Delta\left(i_{0}, \lambda\right) \rightarrow A^{\lambda} .
$$

2.2 Cell modules. We also define modules associated to cells of $\mathcal{B}$. Let $\theta_{X}$ be a cell of $\mathcal{B}$, where $X \in\{L, R, L R\}$ indicates a left, right or two-sided cell. Let $a \in \theta_{X}$ and define

$$
M(\leqslant X a)=\sum_{\substack{b \in \mathcal{B} \\ b \leqslant X}} \mathrm{k} b \text { and } M\left(<_{X} a\right)=\sum_{\substack{b \in \mathcal{B} \\ b \leqslant X a, b \downarrow_{X} a}} \mathrm{k} b .
$$

Both $M\left(\leqslant_{X} a\right)$ and $M\left(<_{X} a\right)$ are left, right or two-sided ideals depending on $X$. We now put $\mathrm{k} \theta_{X}=M\left(\leqslant_{X} a\right) / M\left(<_{X} a\right)$ and call it an $X$-cell module. Let $\Theta_{X}$ be the set of all $X$-cell modules.

(2.2.1) Lemma. (a) Every standard $A$-module $\Delta(\lambda)$ has a $\Theta_{L}$-filtration.

(b) Every standard right $A$-module $\Delta^{\mathrm{op}}(\lambda)$ has a $\Theta_{R}$-filtration.

(c) Every $A^{\lambda}$ has a $\Theta_{L R}$-filtration.

Moreover if $A$ is crystallographic and $f_{\lambda} \neq 0$ for all $\lambda \in \Lambda$, then all $\Delta(\lambda), \Delta^{\mathrm{op}}(\lambda)$ and $A^{\lambda}$ are cell modules. 
Proof. We only prove (a). One may prove (b) and (c) similarly. The last assertion follows from the definition.

By (1.2.4), $\mathcal{B}_{I(\lambda), j}$ is a disjoint union of left cells. Write $\mathcal{B}_{I(\lambda), j}=\bigcup_{i} \Gamma_{i}$ where $\Gamma_{i}$, $1 \leqslant i \leqslant t$, are left cells of $\mathcal{B}$ in $\mathcal{B}_{I(\lambda), j}$. Note that the linear order $\Gamma_{1}, \Gamma_{2}, \cdots$ here is compatible with the induced order $\leqslant_{L}$. For any $i \in[1, t]$ let $M_{i}$ be the $\mathrm{k}$-submodule of $\mathcal{B}_{I(\lambda), j}$ generated by the set $\bigcup_{j=1}^{i} \Gamma_{j}$. Then $M_{i}$ is a left $A$-submodule of $\Delta(\lambda, j)$. It is easy to see that $0=M_{0} \subset M_{1} \subset \cdots \subset M_{t}=\Delta(\lambda, j)$ is a filtration of $\Delta(\lambda, j)$ whose section is isomorphic to a left cell module defined in 2.2. The result follows from (2.1.4).

2.3 The bilinear function $\beta_{\lambda}$. Recall from (1.2.3) the function $f_{\lambda}: J(\lambda) \times I(\lambda) \rightarrow$ $\mathrm{k}$. We define (compare [GL, 2.3]) a bilinear pairing $\beta_{\lambda}: \Delta(\lambda) \times \Delta^{\mathrm{op}}(\lambda) \rightarrow \mathrm{k}$ such that

$$
\beta_{\lambda}\left(a_{i}^{\lambda}, b_{j}^{\lambda}\right)=f_{\lambda}(j, i) .
$$

The notation $\beta_{\lambda}$ should not be confused with the bilinear form $\beta_{\lambda}$ on $A^{\lambda}$. However, we may view $\beta_{\lambda}$ here as the restriction of the $\beta_{\lambda}$ on $A^{\lambda}$ by identifying $\Delta(\lambda)$ and $\Delta^{\mathrm{op}}(\lambda)$ with $\Delta\left(\lambda, j_{0}\right)$ and $\Delta\left(i_{0}, \lambda\right)$ under (2.1.4).

Some of the results related to the function $\beta_{\lambda}$ below are parallel to those in [GL, $\S 2]$ related to the similar function $\phi_{\lambda}$ there.

(2.3.1) Lemma. (a) For any $x \in \Delta(\lambda), y \in \Delta^{\mathrm{op}}(\lambda)$ and $a \in A$, we have

$$
\beta_{\lambda}(a x, y)=\beta_{\lambda}(x, y a) .
$$

(b) If $x, z \in \Delta(\lambda)$ and $y \in \Delta^{\mathrm{op}}(\lambda)$ then $m(x \otimes y) z=\beta_{\lambda}(z, y) x$, where

$$
m: \Delta(\lambda) \otimes_{\mathrm{k}} \Delta^{\mathrm{op}}(\lambda) \rightarrow A^{\lambda}
$$

is the $\mathrm{k}$-module isomorphism given in (2.1.6).

Proof. The statement (a) follows from (2.1.3), (1.2.6) and the previous remark. To prove (b), note that the maps $m$ and $\beta_{\lambda}$ are bilinear and we may assume that $x, y, z$ are standard basis elements in $\Delta(\lambda)$ and $\Delta^{\mathrm{op}}(\lambda)$. Then the verification of (b) is straightforward.

(2.3.2) Corollary. For any $z \in \Delta(\lambda)$, let $I_{z}$ be the ideal of $\mathrm{k}$ defined by

$$
I_{z}=\left\{\beta_{\lambda}(z, y) \mid y \in \Delta^{\mathrm{op}}(\lambda)\right\} .
$$

Then $\Delta(\lambda)$ is A-cyclic if $I_{z}=\mathrm{k}$.

Proof. By (2.3.1)(b) we have

$$
\Delta(\lambda) \supseteq A z \supseteq A^{\lambda} z=\left\{\beta_{\lambda}(z, y) x \mid y \in \Delta^{\mathrm{op}}(\lambda), x \in \Delta(\lambda)\right\}=I_{z} \Delta(\lambda) .
$$

So $I_{z}=\mathrm{k}$ implies $\Delta(\lambda)=I_{z} \Delta(\lambda)$, forcing $\Delta(\lambda)=A z$.

(2.3.3) Corollary. Let $I_{z}=\mathrm{k}$ for some $z \in \Delta(\lambda)$. Then

(a) $\operatorname{Hom}_{A}(\Delta(\lambda), \Delta(\mu))=0$ unless $\lambda \leqslant \mu$,

(b) $\operatorname{Hom}_{A}(\Delta(\lambda), \Delta(\lambda))=\mathrm{k}$.

Proof. Since $I_{z}=\mathrm{k}$, there exists an element $y \in \Delta^{\mathrm{op}}(\lambda)$ such that $\beta_{\lambda}(z, y)=1$, and hence $\Delta(\lambda)=A^{\lambda} z$ by the proof of (2.3.2). Thus $\operatorname{Hom}_{A}(\Delta(\lambda), \Delta(\mu)) \neq 0$ implies that there exist $x \in \Delta(\lambda), a \in A^{\lambda}$ and $f \in \operatorname{Hom}_{A}(\Delta(\lambda), \Delta(\mu))$ such that $a f(x) \neq 0$. Therefore, by (1.2.2), $\lambda \leqslant \mu$. 
Let $\lambda=\mu$. For any $f \in \operatorname{End}_{A}(\Delta(\lambda)), f$ is determined by $z^{\prime}=f(z)$, since $\Delta(\lambda)=A^{\lambda} z$. By (2.3.1)(b) we have

$$
f(z)=f\left(\beta_{\lambda}(z, y) z\right)=f(m(z \otimes y) z)=m(z \otimes y) z^{\prime}=\beta_{\lambda}\left(z^{\prime}, y\right) z .
$$

Thus $f \mapsto \beta_{\lambda}\left(z^{\prime}, y\right)$ gives the isomorphism between $\operatorname{End}_{A}(\Delta(\lambda))$ and $\mathrm{k}$.

(2.3.4) Corollary. Let $\mathrm{k}$ be a field. Then $\Delta(\lambda)$ is A-cyclic if $\beta_{\lambda} \neq 0$.

2.4 Simple modules and projective covers. In this section we assume that $\mathrm{k}$ is a field. Thus a $\mathrm{k}$-algebra $A$ is finite dimensional over $\mathrm{k}$ and an $A$-module is also finite dimensional as a k-space. Recall that, for a given $A$-module $M$, the head hd $(M)$ of $M$ is the largest semisimple quotient module of $M$ and the radical of $M$ is the submodule $\operatorname{rad}(M)$ of $M$ such that $M / \operatorname{rad}(M)=\operatorname{hd}(M)$. If $L$ is a simple $A$-module, let $[M: L]$ be the multiplicity of $L$ in $M$ as a composition factor.

Let $A$ be a finite dimensional standardly based algebra over $\mathrm{k}$. We first determine all non-isomorphic simple modules of $A$.

(2.4.1) Theorem. Let $A$ be a finite dimensional standardly based algebra on $\Lambda$ over a field $\mathrm{k}$ and let $\Lambda_{1}=\left\{\lambda \in \Lambda \mid \beta_{\lambda} \neq 0\right\}$.

(a) For any $\lambda \in \Lambda_{1}$ we have

$$
\operatorname{rad}(\Delta(\lambda))=\left\{v \in \Delta(\lambda) \mid \beta_{\lambda}(v, y)=0, \text { for all } y \in \Delta^{\mathrm{op}}(\lambda)\right\},
$$

and $L(\lambda):=\Delta(\lambda) / \operatorname{rad}(\Delta(\lambda))$ is simple.

(b) Let $\lambda \in \Lambda_{1}$. If $L(\lambda)$ is a composition factor of $\Delta(\mu)$, then $\lambda \leqslant \mu$ and, moreover, $[\Delta(\lambda): L(\lambda)]=1$.

(c) For any $\lambda \in \Lambda_{1}, L(\lambda)$ is absolutely irreducible.

(d) $\left\{L(\lambda) \mid \lambda \in \Lambda_{1}\right\}$ is a complete set of all non-isomorphic simple A-modules.

(e) If $A$ is semisimple, then $L(\lambda)=\Delta(\lambda)$ for all $\lambda \in \Lambda$. Therefore, $A$ is split semisimple.

Proof. Let $R=\left\{v \in \Delta(\lambda) \mid \beta_{\lambda}(v, y)=0\right.$, for all $\left.y \in \Delta^{\mathrm{op}}(\lambda)\right\}$. If $\lambda \in \Lambda_{1}$ then $R \neq \Delta(\lambda)$. Thus, for $0 \neq z \in \Delta(\lambda) / R$, write $z=z_{1}+R$. Since $z_{1} \notin R$, there exists an element $y \in \Delta^{\mathrm{op}}(\lambda)$ such that $\beta_{\lambda}\left(z_{1}, y\right)=1$. So, for any $x \in \Delta(\lambda)$, $x=\beta_{\lambda}\left(z_{1}, y\right) x=m(x \otimes y) z_{1} \in A z_{1}$. This shows that $\Delta(\lambda)=A z_{1}$ and $\Delta(\lambda) / R=$ $A z$, which implies that $\Delta(\lambda) / R$ is a simple left $A$-module. Thus $R \supseteq \operatorname{rad}(\Delta(\lambda))$. If $\operatorname{rad}(\Delta(\lambda)) \neq R$, then there is a simple module $L$ in $\operatorname{hd}(\Delta(\lambda))$ such that the projection $\eta$ from $\Delta(\lambda)$ onto $L$ does not map $R$ to zero. Therefore $\eta(R)=L$. Thus, we have $\eta\left(z_{1}\right)=\eta(u)$ for some $u \in R$, but $\beta(u, y)=0$ as $u \in R$. By (2.3.1(b)), we have

$$
\eta\left(z_{1}\right)=\eta\left(\beta_{\lambda}\left(z_{1}, y\right) z_{1}\right)=m\left(z_{1} \otimes y\right) \eta\left(z_{1}\right)=\eta\left(m\left(z_{1} \otimes y\right) u\right)=\eta\left(\beta(u, y) z_{1}\right)=0 .
$$

So $\eta(\Delta(\lambda))=0$, a contradiction. Therefore $\operatorname{rad} \Delta(\lambda)=R$, and (a) is proved.

We now prove (b). If $L(\lambda)$ is a composition factor of $\Delta(\mu)$, then there is an $A$-homomorphism $\eta: \Delta(\lambda) \rightarrow \Delta(\mu) / N$ for some $A$-submodule $N \subset \Delta(\mu)$ such that $\operatorname{im} \eta \cong L(\lambda)$. Since $\Delta(\lambda)=A^{\lambda} z_{1}$ for some $z_{1} \in \Delta(\lambda)$ as above and $\eta \neq 0$, we have immediately by (1.2.2) that $\mu \geqslant \lambda$. If $\mu=\lambda$ then $\eta$ must be a surjection, since $\eta(z)=z^{\prime}+N=\beta_{\lambda}\left(z_{1}, y\right) z^{\prime}+N=\beta_{\lambda}\left(z^{\prime}, y\right) z+N$. Thus $\Delta(\lambda) / N \cong L(\lambda)$, and hence $[\Delta(\lambda): L(\lambda)]=1$.

By (b) we have $\operatorname{End}_{A}(L(\lambda)) \cong \operatorname{End}_{A}(\Delta(\lambda)) \cong \mathrm{k}$. Therefore, $L(\lambda)$ is absolutely irreducible. 
To prove (d), we first note that $L(\lambda) \cong L(\mu)$ implies $\lambda=\mu$. For any simple $A$-module $L$, we have an epimorphism $f: A \rightarrow L$. Let

$$
0=J_{0} \subset J_{1} \subset \cdots \subset J_{m}=A
$$

be a based filtration given in (1.1.3) with $m=|\Lambda|$. Let $J_{i}$ be the minimal ideal in the above filtration such that $\left.f\right|_{J_{i}} \neq 0$, and put $\lambda=\lambda_{i}$. Then $f$ induces an epimorphism $f: A^{\lambda} \cong J_{i} / J_{i-1} \rightarrow L$ and hence, by (2.1.5), an epimorphism $f: \Delta(\lambda) \rightarrow L$ forcing $L(\lambda) \cong L$ by $(\mathrm{b})$.

Finally, if $A$ is semisimple then any left $A$-module is completely reducible. Thus, $\operatorname{rad}(\Delta(\lambda))=0$, and hence, $L(\lambda)=\Delta(\lambda)$ for any $\lambda \in \Lambda$. Now $\operatorname{End}_{A}(L(\lambda))=\mathrm{k}$ implies that $A$ is split semisimple.

(2.4.2) Corollary. Let $A$ be a standardly full-based algebra on $\Lambda$ over a field $\mathrm{k}$. Then $\Lambda_{1}=\Lambda$, and $\{L(\lambda) \mid \lambda \in \Lambda\}$ is a complete set of all non-isomorphic simple A-modules.

Proof. The assertion follows from Theorem $(2.4 .1)(\mathrm{d})$ and the definition of a standardly full-based algebra.

Note that if $\Lambda_{1}$ happens to be an ideal, then $A / A\left(\Lambda \backslash \Lambda_{1}\right)$ is a standardly fullbased algebra on the poset $\Lambda_{1}$.

(2.4.3) Lemma. Let $A$ be a standardly based algebra on $\Lambda$ and let ${ }_{A} P$ be a projective indecomposable submodule of $A$. If $\lambda \in \Lambda$ is a minimal element such that $A^{\lambda} P \neq 0$, then $\lambda \in \Lambda_{1}$ and $P$ is the projective cover of $L(\lambda)$. Moreover, we have $P=A^{\geqslant \lambda} P$.

Proof. Let $\Gamma$ be the coideal of $\Lambda$ generated by $\left\{\mu \in \Lambda \mid A^{\mu} P \neq 0\right\}$. Then the ideal $A(\Gamma)$ of $A$ defined in 1.1 has the property that $A(\Gamma) \otimes_{A} P \cong A(\Gamma) P=P$, and so $A^{\lambda} P$ is a homomorphic image of $P$. Now

$$
\begin{aligned}
\operatorname{Hom}_{A}(P, \Delta(\lambda)) & \supseteq \operatorname{Hom}_{A}\left(A^{\lambda} \otimes_{A} P, \Delta(\lambda)\right) \\
& \cong \operatorname{Hom}_{\mathrm{k}}\left(\Delta^{\mathrm{op}}(\lambda) \otimes_{A} P, \operatorname{End}_{A}(\Delta(\lambda)) \quad \text { by }(2.1 .4)\right. \\
& \cong \operatorname{Hom}_{\mathrm{k}}\left(\Delta^{\mathrm{op}}(\lambda) \otimes_{A} P, \mathrm{k}\right) \quad \text { by }(2.3 .3) \\
& \neq 0 .
\end{aligned}
$$

So, if $\operatorname{hd}(P)=L(\nu)$ for some $\nu \in \Lambda_{1}$, then $L(\nu)$ is a composition factor of $\Delta(\lambda)$. By $(2.4 .1)(\mathrm{b})$ we have $\nu \leqslant \lambda$. However, since $\Delta(\nu)$ is a homomorphic image of $P$ and $\Delta(\nu)=A^{\nu} \Delta(\nu)$ (see (2.3.2)), it follows that $A^{\nu} P \neq 0$. Therefore $\nu \geqslant \lambda$, and hence $\nu=\lambda$. Now $\lambda$ is the unique minimal element of $\Gamma$, and the last assertion follows.

We shall denote the projective cover of $L(\lambda)$ by $P(\lambda)$, and let

$$
\Delta=\{M \in \mathrm{Ob}(A \text {-mod }) \mid M \cong \Delta(\lambda) \text { for some } \lambda \in \Lambda\} .
$$

(2.4.4) Proposition. Let $A$ be a standardly based algebra. For $\lambda \in \Lambda_{1}$, the projective cover $P(\lambda)$ has a $\Delta$-filtration. If $[P(\lambda): \Delta(\mu)]$ denotes the number of sections isomorphic to $\Delta(\mu)$ in such a filtration, then $[P(\lambda): \Delta(\mu)] \neq 0 \Rightarrow \mu \geqslant \lambda$ and $[P(\lambda): \Delta(\lambda)]=1$.

Proof. We have by (2.4.3) $P(\lambda)=A^{\geqslant \lambda} P(\lambda) \cong A^{\geqslant \lambda} \otimes_{A} P(\lambda)$. We order $\Lambda^{\geqslant \lambda}=$ $\left\{\lambda_{1}, \cdots, \lambda_{t}=\lambda\right\}$ in the same manner as in (1.1.2). Thus, we have a filtration of $A^{\geqslant \lambda}$ as in (1.1.3) with $t=m$. Tensoring this filtration with $P(\lambda)$ gives a filtration of 
$P(\lambda)$ by the projectivity of $P(\lambda)$. Now the sections of this filtration are of the form $A^{\lambda_{i}} \otimes_{A} P(\lambda)(1 \leqslant i \leqslant t)$, which is isomorphic to a direct sum of $\Delta\left(\lambda_{i}\right)$ by $(2.1 .5)$, and $A^{\lambda} \otimes_{A} P(\lambda) \cong \Delta(\lambda)$ by the proof of (2.4.3). The result follows easily.

(2.4.5) Definition. Let $A$ be a standardly based algebra on a poset $\Lambda$ over a discrete valuation ring $\mathcal{O}$ with quotient field $K$ and residue field $\mathrm{k}$, and assume that $A_{K}$ is semisimple. For any $\lambda, \mu \in \Lambda$, let $d_{\lambda, \mu}$ be the multiplicity of $L(\mu)$ in $\Delta(\lambda)$. Then $D=\left(d_{\lambda, \mu}\right)_{\substack{\lambda \in \Lambda \\ \mu \in \Lambda_{1}}}$ is said to be the decomposition matrix of $A$.

By Theorem (2.4.1), we have the following proposition.

(2.4.6) Proposition. Keep the notation introduced in (2.4.5). Let $A$ be a standardly based algebra over $\mathcal{O}$. Then the decomposition matrix of $A$ is upper unitriangular.

\section{QUASI-HEREDITY OF STANDARDLY FULL-BASED ALGEBRAS}

In this and the next section, we shall prove the main results of the paper. We first prove that standardly full-based algebras are in fact split quasi-hereditary algebras. Then we shall prove in the next section that every split quasi-hereditary algebra is a standardly full-based algebra. Throughout, $\mathrm{k}$ denotes a commutative noetherian ring unless otherwise specified.

3.1 Quasi-hereditary algebras. Quasi-hereditary algebras are a class of finite dimensional algebras which were first introduced by E. Cline, B. Parshall and L. Scott in order to deal with highest weight categories arising from the representations of Lie algebras and algebraic groups. However, the importance of quasi-hereditary algebras lies also in ring theory itself. Many interesting algebras such as Auslander algebras and Schur algebras turn out to be quasi-hereditary. The definition below, taken from [CPS3], is about integral quasi-hereditary algebras, i.e., quasi-hereditary algebras over a commutative noetherian ring k. Following [CPS3], we shall only consider $\mathrm{k}$-algebras $A$ which are finitely generated as $\mathrm{k}$-modules, i.e., $k$-finite algebras. Recall from [CPS3] that $A$ is k-semisimple if, for any $A$-module $M$, the natural projection $A \otimes_{\mathrm{k}} M \rightarrow M$ is $A$-split.

(3.1.1) Definition. An ideal $J$ in a k-projective algebra $A$ is said to be a heredity ideal if $A / J$ is k-projective and the following conditions hold.

(a) $J$ is projective as a left $A$-module.

(b) $J^{2}=J$.

(c) The k-algebra $E=\operatorname{End}_{A}\left({ }_{A} J\right)$ is k-semisimple.

A k-projective algebra $A$ is called to be a quasi-hereditary algebra if there is a sequence

$$
0=J_{0} \subset J_{1} \subset \cdots \subset J_{t}=A
$$

of ideals in $A$ such that $J_{i} / J_{i-1}$ is a heredity ideal in $A / J_{i-1}$ for $i=1,2, \ldots, t$. We shall call such a sequence a heredity chain.

Note that if $\mathrm{k}$ is a field, then (c) is equivalent to

$\left(\mathrm{c}^{\prime}\right) J \cdot \operatorname{rad}(A) \cdot J=0($ see $[$ CPS3] $)$.

Thus (3.1.1) agrees with the definition for quasi-hereditary algebras over fields in [CPS1]. Also, we adopt definitions for the quasi-hereditary algebras of separable and split types over a commutative noetherian ring given in [CPS3]. 
An alternative way to describe quasi-hereditary algebras $A$ over fields is the notion of highest weight categories defined in [CPS1], where it is proved that $A$ is a quasi-hereditary algebra if and only if $A$-mod is a highest weight category. A highest weight category $\mathcal{C}$ is a certain abelian category defined on a weight poset $\Lambda$ satisfying (a) The non-isomorphic simple object in $\mathcal{C}$ are indexed as $L(\lambda), \lambda \in \Lambda$; (b) For any $\lambda \in \Lambda$, there is a standard object $\Delta(\lambda)$ whose composition factors are of form $L(\mu)$ with $\mu \leqslant \lambda$, and moreover, $L(\lambda)$ occurs with multiplicity one; (c) The projective cover $P(\lambda)$ of $L(\lambda)$ has a $\Delta$-filtration and $\Delta(\lambda)$ occurs with multiplicity one. This definition has been generalized by taking up $\mathrm{k}$ to a commutative local noetherian ring in [DS, 2.1], where the notion of $\mathrm{k}$-finite highest weight category is introduced. Thus, if we denote by $\mathfrak{m}$ the maximal ideal of $\mathrm{k}$, by $\overline{\mathrm{k}}$ the residue field and by $\bar{M}$ the reduction modulo $\mathfrak{m}$ of any $\mathrm{k}$-module $M$, then, in a $\mathrm{k}$-finite highest weight category $\mathcal{C}$, the standard $\mathrm{k}$-free objects $\Delta(\lambda)$ exist and their reductions modulo $\mathfrak{m}$ are the standard objects of the full subcategory $\overline{\mathcal{C}}$ of objects $\bar{M}$ with $M$ in $\mathcal{C}$, which is a highest weight category over the field $\overline{\mathrm{k}}$. We have the following (see [DS, 2.1.1, 2.3, 2.5]).

(3.1.2) Theorem. Let $\mathrm{k}$ be a commutative local noetherian ring. A $\mathrm{k}$-finite algebra is quasi-hereditary of separable type if and only if the category $\mathcal{C}=A$ - $\bmod$ of $\mathrm{k}$ finite A-modules is a $\mathrm{k}$-finite highest weight category with a finite weight poset $\Lambda$ and all $\operatorname{End}_{\mathcal{C}}(L(\lambda))$ being $\overline{\mathrm{k}}$-separable. Moreover, the projective cover $P(\lambda)$ of $L(\lambda)$, filtered by standard modules, exists.

(3.1.3) Proposition. Keep the notation in (3.1.2). There is a one to one correspondence between the set of compatible linear orders for $\Lambda$ as defined in (1.1.2) and the set of longest heredity chains of $A$.

Proof. Let $P(\lambda)$ be the projective cover of $L(\lambda)$ (see [DS, 2.4]) and $l_{\lambda}=\operatorname{dim}_{\overline{\mathrm{k}}} L(\lambda)$. If $P=\bigoplus_{\lambda \in \Lambda} P(\lambda)^{\oplus l_{\lambda}}$, then we may identify $A$ with the algebra $\operatorname{End}_{A}(P)^{\mathrm{op}}$.

Let $\Lambda=\left\{\lambda_{1}, \lambda_{2}, \cdots, \lambda_{m}\right\}$ be a compatible linear order and $P_{i}=\bigoplus_{j \leq i} P\left(\lambda_{j}\right)$. Then, one sees easily that $\operatorname{Hom}_{A}\left(P, P_{i}\right)=A e_{i}$, where $e_{i}=\operatorname{id}_{P_{i}}$ is viewed as an element of $A$. Let

$$
J_{i}=\operatorname{Hom}_{A}\left(P, P_{i}\right) \operatorname{Hom}_{A}\left(P_{i}, P\right)=A e_{i} A .
$$

Then, by the proof of [DS, 2.5], the sequence

$$
0=J_{0} \subseteq J_{1} \subseteq \cdots \subseteq J_{m}=A
$$

is a heredity chain of $A$. To show the uniqueness, it suffices by induction to show that if $J$ is any heredity ideal of $A$ with $J \cong P\left(\lambda_{1}\right)^{\oplus r}$, then $J=J_{1}$. This can be shown as follows. Write $J=P_{1} \oplus \cdots \oplus P_{r}$, where $P_{i} \cong A e_{1}$. So, if $f_{i}: A e_{1} \rightarrow P_{i}$ is the isomorphism, then $P_{i}=f_{i}\left(A e_{1}\right)=A e_{1} f_{i}\left(e_{1}\right) \subseteq A e_{1} A$. Therefore, $J \subseteq A e_{1} A=J_{1}$. Conversely, from the projectivity of $J$, there is an idempotent $e^{\prime} \in A$ such that $P_{1}=A e^{\prime}$, and so $A e^{\prime} A \subseteq J$. Reversing the roles of $J$ and $J_{1}$ in the previous proof, we obtain $J_{1} \subseteq A e^{\prime} A$. Therefore, $J=J_{1}$.

From the proof above, we conclude the following.

(3.1.4) Corollary. If $J$ is a heredity ideal for which the head of $\bar{J}$ is isomorphic to a direct sum of a single $L(\lambda)$ with $\lambda \in \Lambda$ maximal, then

$$
J=\sum_{M \leqslant A, M \cong P(\lambda)} M .
$$


3.2. Quasi-heredity of standardly full-based algebras. Let $\operatorname{Spec}(\mathrm{k})$ be the set of all prime ideals of $\mathrm{k}$. For $\mathfrak{p} \in \operatorname{Spec}(\mathrm{k})$, recall the notation introduced before (1.3.2): $\mathrm{k}(\mathfrak{p})=k_{\mathfrak{p}} / \mathfrak{p}_{\mathfrak{p}}, M(\mathfrak{p})=M \otimes_{\mathrm{k}} \mathrm{k}(\mathfrak{p})$ and $\bar{J}(\mathfrak{p})$, where $M$ is a k-module and $J$ is an ideal of $A$. Note that $\bar{J}(\mathfrak{p})$ denotes the image of $J(\mathfrak{p})$ in $A(\mathfrak{p})$.

(3.2.1) Theorem. Let $\mathrm{k}$ be a commutative noetherian ring and let $A$ be a standardly full-based algebra over $\mathrm{k}$. Then $A$ is quasi-hereditary over $\mathrm{k}$. If, in addition, $\mathrm{k}$ is local, then $A$ is split quasi-hereditary.

Proof. Let

$$
0=J_{0} \subset J_{1} \subset \cdots \subset J_{m}=A
$$

be a based filtration of $A$ as given in (1.1.3). Thus, $J:=J_{1}$ is generated by $\mathcal{B}^{\lambda}$, where $\lambda=\lambda_{1}$ in the notation of (1.1.3). It is easy to see that $A / J$ is a standardly based algebra on the poset $\Lambda \backslash\{\lambda\}$ with the image of $\mathcal{B} \backslash \mathcal{B}^{\lambda}$ as defining basis. Thus, to show that (1.1.3) is a heredity chain of $A$ and $A$ is split, it suffices to show that $J$ is a heredity ideal of $A$ of split type. However, to show that $J$ is a heredity ideal is also equivalent to proving that $\bar{J}(\mathfrak{p})$ is a heredity ideal of $A(\mathfrak{p})$ for all $\mathfrak{p} \in \operatorname{Spec}(\mathrm{k})$ (see [CPS3, 3.3]).

For $\mathfrak{p} \in \operatorname{Spec}(\mathrm{k})$, one sees easily that $A(\mathfrak{p})$ is a standardly based $\mathrm{k}(\mathfrak{p})$-algebra, since $A$ is $\mathrm{k}$-free and

$$
0=\bar{J}_{0}(\mathfrak{p}) \subset \bar{J}_{1}(\mathfrak{p}) \subset \cdots \subset \bar{J}_{m}(\mathfrak{p})=A(\mathfrak{p})
$$

is a based filtration of $A(\mathfrak{p})$. By (2.1.4), we have $\bar{J}(\mathfrak{p}) \cong \Delta(\lambda)^{\oplus r_{\lambda}}$. Here, for simplicity of notation, $\Delta(\lambda)$ denotes the standard module of $A(\mathfrak{p})$. Since $\beta_{\lambda}(\mathfrak{p}) \neq 0$, $\operatorname{End}_{A(\mathfrak{p})}(J(\mathfrak{p}))$ is isomorphic to the ring of full $r_{\lambda} \times r_{\lambda}$ matrices over the field $\mathrm{k}(\mathfrak{p})$ by (2.3.3). Hence, it is a semisimple $\mathrm{k}(\mathfrak{p})$-algebra.

As shown above, $\bar{J}(\mathfrak{p})$ is an ideal of $A(\mathfrak{p})$ and $\bar{J}(\mathfrak{p}) \cong \Delta(\lambda)^{\oplus r_{\lambda}}$. Using $\beta_{\lambda}(\mathfrak{p}) \neq 0$ again, we see that $\Delta(\lambda)$ has a simple head $L(\lambda)$. Let $P(\lambda)$ be the projective cover of $L(\lambda)$. Then, by (2.4.4) and the maximality of $\lambda$, we have $P(\lambda)=\Delta(\lambda)$, and hence $\bar{J}(\mathfrak{p})$ is projective as left $A(\mathfrak{p})$-module.

Finally, by (1.3.2), we have $J^{2}=J$. Thus $\bar{J}(\mathfrak{p})^{2}=\bar{J}(\mathfrak{p})$ for all $\mathfrak{p} \in$ Speck. So we have proved that $\bar{J}(\mathfrak{p})$ is a heredity ideal of $A(\mathfrak{p})$ for all $\mathfrak{p} \in$ Spec $\mathrm{k}$. By the remark at the beginning of the proof, $A(\mathfrak{p})$ is quasi-hereditary, and hence so is $A$.

Assume now $\mathrm{k}$ is local. Then there must be $x, y \in \mathcal{B}^{\lambda}$ such that $\beta_{\lambda}(x, y)$ is invertible in $\mathrm{k}$, since $\operatorname{im}\left(\beta_{\lambda}\right)=\mathrm{k}$ is generated by the image of $f_{\lambda}$. This implies that $(2.3 .3(\mathrm{~b}))$ holds. Consequently, the algebra $\operatorname{End}_{A}(J)$ is isomorphic to a full matrix algebra over $\mathrm{k}$ and, therefore, split.

(3.2.2) Corollary. Let $A$ be a standardly full-based algebra over a commutative local noetherian ring $\mathrm{k}$.

(a) Every based filtration defined in (1.1.3) of $A$ is a heredity chain of A. Therefore, based filtrations exhaust all the longest heredity chains of $A$ by (3.1.3).

(b) The standard and costandard modules defined in (2.1.2) agree with their counterparts in the $\mathrm{k}$-finite highest weight category A-mod.

(c) If $\mathcal{B}^{\prime}$ is another standard defining base of $A$ defined with respect to another partial order $\leqslant$ on $\Lambda$, then $A$ must be full with respect to $\mathcal{B}^{\prime}$.

Proof. To see (c), we note that the reduction $\bar{A}$ modulo $\mathfrak{m}$ is standardly based with respect to $\left(\Lambda, \leqslant^{\prime}\right)$ and $\overline{\mathcal{B}}^{\prime}$. Thus, if $\Lambda_{1}$ is defined with respect to $\overline{\mathcal{B}}^{\prime}$ as in (2.4.1), 
then we must have $\Lambda_{1}=\Lambda$ as $\Lambda$ indexes the simple $\bar{A}$-modules, forcing $\operatorname{im}\left(\beta_{\lambda}\right)=\mathrm{k}$ for all $\lambda \in \Lambda$. Therefore, $A$ is standardly full-based with respect to $\mathcal{B}^{\prime}$.

(3.2.3) Remark. (a) Result (3.2.2) gives the following property of a standard defining base: If $A$ is standardly full-based algebra (hence quasi-hereditary) with defining base $\mathcal{B}$, fibred over a poset $\Lambda$, and

$$
0=I_{0} \subset I_{1} \subset \cdots \subset I_{t}=A
$$

is a heredity chain of $A$, then every ideal $I_{j}$ has a basis of the form

$$
\mathcal{B}^{\Gamma}=\bigcup_{\lambda \in \Gamma} \mathcal{B}^{\lambda} \quad \text { for some coideal } \Gamma \subseteq \Lambda .
$$

We shall call this property the global property of the defining base $\mathcal{B}$.

(b) Let $A$ be a standardly full-based algebra over a field $\mathrm{k}$. Then, by using the equivalence between quasi-hereditary algebras and highest weight categories (cf. [CPS1]), the result (3.2.2) is equivalent to the fact that the category $A$-mod is a highest weight category with weight poset $\Lambda$. Thus we can get another proof of (3.2.2). In this approach, the standard module $\Delta(\lambda)$ and costandard module $\nabla(\lambda)$ defined in (2.1.2) are the standard module and costandard module in the highest weight category $A$-mod. Result (2.4.1) gives the required condition on the composition factors of $\Delta(\lambda)$, and the condition on the $\Delta$-filtration for $P(\lambda)$ is given in (2.4.4).

(c) When $A$ is cellular and full over a field, the quasi-heredity of $A$ has been mentioned without proof in [GL, 3.10].

(3.2.4) Example. By Theorem (3.2.1), the Borel subalgebras $S^{\preccurlyeq}$ and $S^{\succcurlyeq}$ described in (1.3.4) are integrally quasi-hereditary.

\section{Standard BASES FOR SPlit QUASI-HEREDitary ALGEBRAS}

We are going to prove the converse of Theorem (3.2.1). Throughout this section, $\mathrm{k}$ denotes a commutative local noetherian ring.

4.1. Shuffling heredity chains. Let $A$ be a quasi-hereditary algebra over $\mathrm{k}$ with weight poset $\Lambda$. Let $\lambda \in \Lambda$ be a fixed maximal element. By (3.1.4) there is a heredity ideal $J$ associated to $\lambda$. Thus the quotient algebra $B=A / J$ is quasi-hereditary over k with weight poset $\Lambda \backslash\{\lambda\}$. Let $\pi: A \rightarrow B$ be the natural projection.

A longest heredity chain, say

$$
0=J_{0} \subset J_{1} \subset \cdots \subset J_{m}=A,
$$

determines a compatible linear order of $\Lambda: \lambda_{1}, \lambda_{2}, \cdots$. When $\lambda_{1} \neq \lambda$ we want to shuffle (4.1.1) to obtain a heredity chain

$$
0=I_{0} \subset I_{1} \subset \cdots \subset I_{m}=A
$$

such that $I_{1}=J$ and the images of (4.1.1) and (4.1.2) in $B$ are the same.

Let $\Delta(\nu)$ (resp. $\left.\Delta^{\mathrm{op}}(\nu)\right)$ be the standard module corresponding to $\nu \in \Lambda$ in the category of k-finite left (resp. right) $A$-modules.

The following lemma is necessary when shuffling a heredity chain (see [DS, 2.2]).

(4.1.3) Lemma. Let $\mathrm{k}$ be a commutative local noetherian ring. Let $A$ be a quasihereditary $\mathrm{k}$-algebra of separable type with the poset $\Lambda$. Then

$$
\operatorname{Ext}_{A}^{1}(\Delta(\lambda), \Delta(\mu))=0
$$

unless $\lambda<\mu$. 
(4.1.4) Proposition. Let $\mathrm{k}$ be a commutative local noetherian ring. Let $A$ be a separable quasi-hereditary $\mathrm{k}$-algebra. Suppose $\lambda \in \Lambda$ is maximal and $J$ is the associated heredity ideal. Let $B=A / J$, and let $\pi$ be the natural homomorphism $A \rightarrow B$. If (4.1.1) is a heredity chain of $A$ of maximal length and $l$ is the smallest number such that $J \subseteq J_{l}$, then

(a) $J_{l}=J_{l-1}+J=J_{l-1} \oplus J$,

(b) the sequence

$$
0=\pi\left(J_{0}\right) \subseteq \pi\left(J_{1}\right) \subseteq \cdots \subseteq \pi\left(J_{l-1}\right) \subseteq \pi\left(J_{l+1}\right) \subseteq \cdots \subset \pi\left(J_{m}\right)=B
$$

is a heredity chain of $B$, and

(c) the sequence

$$
0 \subset J \subset J_{1} \oplus J \subset \cdots \subset J_{l-1} \oplus J \subset J_{l+1} \subset \cdots \subset J_{m}=A
$$

is a heredity chain of $A$.

Proof. Let (1.1.2) be the compatible linear order corresponding to the given heredity chain (4.1.1) and let $\lambda=\lambda_{i_{0}}$. Since $\lambda$ is maximal and every $J_{i}$ has a $\Delta$ filtration, by (4.1.3) and a short-long exact sequence argument we have $J_{i_{0}}=$ $J_{i_{0}-1} \oplus J_{i_{0}} / J_{i_{0}-1}$. Thus, $J_{i_{0}} / J_{i_{0}-1}$ is a heredity ideal of $A$ for which the head of $\bar{J}_{i_{0}} / \bar{J}_{i_{0}-1}$ is a direct sum of $L(\lambda)$. Therefore, (3.1.4) implies $J=J_{i_{0}} / J_{i_{0}-1}$ and hence $l=i_{0}$. So the statement (a) is proved.

By (a), we see that

$$
\pi\left(J_{i}\right) \cong \begin{cases}J_{i}, & \text { for all } i<l, \\ J_{i} / J & \text { for all } i>l,\end{cases}
$$

and $\pi\left(J_{l-1}\right)=\pi\left(J_{l}\right)$. It is straightforward to verify that the given sequence in (b) is a heredity chain of $B$ (cf. [DS, 2.8]).

Finally, (c) follows immediately from (b).

So the sequence given in part (c) is the required sequence (4.1.2).

4.2 Split quasi-hereditary algebras. We need also several facts about split quasi-hereditary algebras. We first note the following.

(4.2.1) Lemma. Let $\mathrm{k}$ be a commutative local noetherian ring. Let $A$ be a quasihereditary algebra with weight poset $(\Lambda, \leqslant)$. If $\mathcal{B}$ is a standard defining base of $A$ on $\left(\Lambda, \leqslant^{\prime}\right)$, where $\leqslant^{\prime}$ is possibly different from $\leqslant$, then $A$ is standardly full-based and split.

Proof. This follows immediately from a similar argument for (3.2.2)(c).

(4.2.2) Proposition. Let $\mathrm{k}$ be a commutative local noetherian ring. Let $A$ be a split quasi-hereditary algebra with weight poset $\Lambda$.

(a) For any $\lambda \in \Lambda$, let $\Delta(\lambda)$ be the standard module in the category A-mod. Then $\operatorname{End}_{A}(\Delta(\lambda)) \cong \mathrm{k}$.

(b) If $\lambda \in \Lambda$ is maximal then $J \cong \Delta(\lambda) \otimes_{\mathrm{k}} \Delta^{\mathrm{op}}(\lambda)$ as bimodule, where $J$ is the heredity ideal corresponding to $\lambda$.

Proof. (a) This follows from the discussion at the end of [CPS3]. For completeness, we include a proof. 
Let $0=J_{0} \subset J_{1} \subset \cdots \subset J_{m}$ be a heredity chain of $A$ of maximal length. Thus each $E_{i}=\operatorname{End}_{A / J_{i-1}}\left(J_{i} / J_{i-1}\right)$ is split over $\mathrm{k}$ (and hence is a direct sum of full matrix rings over $\mathrm{k}$, since $\mathrm{k}$ is local [CPS3, 2.4]). Thus every standard module $\Delta(\lambda)$ is of the form $J_{i} / J_{i-1} \otimes_{E_{i}} P$ for some indecomposable direct summand $P$ of $E_{i}$. So we have

$$
\begin{aligned}
\operatorname{End}_{A}(\Delta(\lambda)) & \cong \operatorname{Hom}_{E_{i}}\left(P, \operatorname{Hom}_{A}\left(J_{i} / J_{i-1}, J_{i} / J_{i-1} \otimes_{E_{i}} P\right)\right) \\
& \cong \operatorname{Hom}_{E_{i}}\left(P, \operatorname{Hom}_{A}\left(J_{i} / J_{i-1}, J_{i} / J_{i-1}\right) \otimes_{E_{i}} P\right) \\
& \cong \operatorname{Hom}_{E_{i}}(P, P) \cong \mathrm{k} .
\end{aligned}
$$

(b) Since $J=A e A$ and $J$ is projective, the multiplication map

$$
m: A e \otimes_{e A e} e A \rightarrow A e A
$$

is bijective by [DR1, Statement 7]. Since $A e \cong P(\lambda)=\Delta(\lambda)$ and

$$
e A e \cong \operatorname{End}_{A}(A e)^{\mathrm{op}} \cong \mathrm{k},
$$

we have $J \cong \Delta(\lambda) \otimes_{\mathrm{k}} \Delta^{\mathrm{op}}(\lambda)$, where $\Delta^{\mathrm{op}}(\lambda) \cong e A$.

We are now ready to prove the following.

(4.2.3) Theorem. Let $\mathrm{k}$ be a commutative local noetherian ring and let $A$ be a split quasi-hereditary algebra over $\mathrm{k}$ with weight poset $\Lambda$. Then $A$ is a standardly full-based algebra.

Proof. We apply induction on $|\Lambda|$. If $\Lambda=\{\lambda\}$ then we have, by (4.2.2), a bimodule isomorphism $A \cong \Delta(\lambda) \otimes_{\mathrm{k}} \Delta^{\mathrm{op}}(\lambda)$. If $\left\{a_{i}^{\lambda}\right\}$ and $\left\{b_{j}^{\lambda}\right\}$ are fixed bases for $\Delta(\lambda)$ and $\Delta^{\mathrm{op}}(\lambda)$ respectively, and $a_{i j}^{\lambda}$ is the image of $a_{i}^{\lambda} \otimes b_{j}^{\lambda}$ in $A$, then one sees easily that $\left\{a_{i j}^{\lambda}\right\}$ is a standard defining base.

Assume $|\Lambda|>1$ and let $\lambda \in \Lambda$ be a maximal element. Let $\lambda=\lambda_{1}, \lambda_{2}, \ldots, \lambda_{n}$ be a compatible linear order for $\Lambda$ such that $\left\{\lambda_{2}, \lambda_{3}, \ldots, \lambda_{t}\right\}=\Lambda^{\prime}=\left\{\mu \in \Lambda_{B} \mid \mu \nless \lambda\right\}$, where $\Lambda_{B}=\Lambda \backslash\{\lambda\}$. Then, by (3.1.3), there is a heredity chain of $A$ associated to this order for $\Lambda$,

$$
0=J_{0} \subset J_{1} \subset \cdots \subset J_{m}=A .
$$

Thus, by (4.2.2), $J=J_{1} \cong \Delta(\lambda) \otimes_{\mathrm{k}} \Delta^{\mathrm{op}}(\lambda)$. Let $B=A / J$, and $\pi: A \rightarrow B$ the natural epimorphism. Then $B$ is a quasi-hereditary algebra with weight poset $\Lambda_{B}$. By induction, $B$ is standardly (full-)based with defining base $\left\{b_{i j}^{\mu}\right\}$. So it has the global property as described in (3.2.3)(a). In particular, $\left\{b_{i j}^{\mu} \mid \mu \in \Lambda^{\prime},(i, j) \in\right.$ $I(\mu) \times J(\mu)\}$ is a basis for $J_{t} / J$. By (4.1.4), we have $J_{t} \cong J_{t} / J \oplus J$. So $J_{t} / J$ can be viewed as an ideal of $A$. Thus, for any $\mu \in \Lambda$, we define

$$
a_{i j}^{\mu}= \begin{cases}b_{i j}^{\mu}, & \text { if } \mu \in \Lambda^{\prime}, \\ c_{i j}^{\mu}, & \text { if } \mu \in \Lambda_{B} \backslash \Lambda^{\prime}, \\ a_{i j}^{\lambda}, & \text { if } \mu=\lambda,\end{cases}
$$

where $c_{i j}^{\mu}$ is an inverse image of $b_{i j}^{\mu}$ and $a_{i j}^{\lambda}$ is defined via the isomorphism $J \cong$ $\Delta(\lambda) \otimes_{\mathrm{k}} \Delta^{\mathrm{op}}(\lambda)$ as above.

We claim that $A$ is a standardly based algebra with $\left\{a_{i j}^{\lambda}\right\}$ as a defining base, and therefore $A$ must be full by (4.2.1). Indeed, it is easy to see that (1.2.2) holds if $\mu=\lambda$. If $\mu \neq \lambda$, then either $\mu \in \Lambda^{\prime}$ or $\mu \in \Lambda_{B} \backslash \Lambda^{\prime}$. In the first case, (1.2.2) 
follows by induction and the fact $J_{t} \cong J_{t} / J \oplus J$ by (4.1.4). In the second case, where $\mu \in \Lambda_{B} \backslash \Lambda^{\prime}$, we have for $a \in A$

$$
\pi(a) \cdot b_{i j}^{\mu} \equiv \sum_{i^{\prime}} f_{i^{\prime}, \mu}(i, \pi(a)) b_{i^{\prime} j}^{\mu} \quad \bmod \left(B^{>\mu}\right), \quad \text { and similarly for } b_{i j}^{\mu} \cdot \pi(a)
$$

by the induction hypothesis on the basis $\left\{b_{i, j}^{\mu}\right\}$ of $B$. Thus we have

$$
a \cdot c_{i j}^{\mu} \equiv \sum_{i^{\prime}} f_{i^{\prime}, \mu}(i, \pi(a)) c_{i^{\prime} j}^{\mu} \quad \bmod \left(A^{>\mu}\right), \quad \text { and similarly for } c_{i j}^{\mu} \cdot a,
$$

since $J \subset A^{>\mu}$ is an ideal by assumption. Hence we have proved that the basis defined inductively in (4.2.4) is a standard defining base of $A$.

It is possible by taking inverse images of certain idempotents as basis elements to construct a standard defining base in the proof above satisfying

$$
a_{i j}^{\lambda} a_{i j}^{\lambda} \equiv a_{i j}^{\lambda} \quad \bmod \left(A^{>\lambda}\right), \text { for some } i, j .
$$

Thus, the fullness follows automatically.

We remark that the construction in the proof depends on the selection of the bases for the standard modules $\Delta(\lambda)$ and $\Delta^{\mathrm{op}}(\lambda)$. However, if we fix, for each $\lambda \in \Lambda$, bases $\left\{a_{i}^{\lambda} \mid i \in I(\lambda)\right\}$ and $\left\{b_{j}^{\lambda} \mid j \in J(\lambda)\right\}$ for $\Delta(\lambda)$ and $\Delta^{\mathrm{op}}(\lambda)$, respectively, then any two defining bases for $A$ defined inductively by (4.2.4) are equivalent.

(4.2.5) Definition. We shall call the union

$$
\bigcup_{\lambda \in \Lambda}\left\{a_{i}^{\lambda}, b_{j}^{\lambda} \mid i \in I(\lambda), j \in J(\lambda)\right\}
$$

a local basis for standard modules, and we shall call a standard defining base of a quasi-hereditary algebra $A$ a (global) standard basis of $A$.

From the previous remark, one has clearly the following.

(4.2.6) Corollary. Let $A$ be a split quasi-hereditary algebra over a commutative local noetherian ring. Then there is a surjective map from the set of local bases for standard modules to the set of equivalence classes of standard bases for A.

Proof. The surjectivity follows from (2.1.4).

So we may introduce an equivalence relation on the set of local bases for standard modules so that the equivalence classes correspond bijectively to the equivalence classes of standard bases of $A$.

We also remark that corollary (4.2.6) can be viewed as a generalization of the relationship between the global canonical bases for the modified quantized enveloping algebras and the local canonical bases for the irreducible representations (see $[\mathrm{Lu}$, Part 4]).

Combining (4.2.3) with (3.2.1), we obtain the main result of the paper.

(4.2.7) Theorem. Let $\mathrm{k}$ be a commutative local noetherian ring. Then $A$ is a split quasi-hereditary algebra over $\mathrm{k}$ if and only if $A$ is a standardly full-based algebra. In particular, if $\mathrm{k}$ is an algebraically closed field, then a finite dimensional $\mathrm{k}$-algebra is quasi-hereditary if and only if it is standardly full-based. 
4.3 An application. We assume that $\mathrm{k}$ is a field in this subsection. Let $A$ be a finite dimensional algebra over $\mathrm{k}$. Recall that $A$ is said to be split over $\mathrm{k}$ if $\operatorname{End}_{A}(L) \cong \mathrm{k}$ for every simple $A$-module $L$. We shall prove that a non-semisimple split symmetric algebra $A$ over $\mathrm{k}$ is not quasi-hereditary. This result is known by a consideration of the global dimension of $A$. The proof below is just a simple application of our theory about a standard basis of $A$.

Recall that a finite dimensional algebra $A$ over $\mathrm{k}$ is said to be symmetric if $A$ admits a non-degenerate bilinear form $\phi: A \times A \rightarrow \mathrm{k}$ which is associative:

$$
\phi(a b, c)=\phi(a, b c), \quad a, b, c \in A,
$$

and symmetric:

$$
\phi(a, b)=\phi(b, a) \quad a, b \in A .
$$

(4.3.1) Theorem. Let $A$ be a finite dimensional split k-algebra. If $A$ is symmetric and non-semisimple, then $A$ is not quasi-hereditary.

Proof. Suppose $A$ is quasi-hereditary. Then, by Theorem (4.2.1), $A$ is a standardly full-based algebra on the weight poset $\Lambda$. Let

$$
\mathcal{B}=\left\{a_{i j}^{\lambda} \mid \lambda \in \Lambda, i \in I(\lambda), j \in J(\lambda)\right\}
$$

be a standard defining basis of $A$. Since $A$ is symmetric with the associated symmetric form $\phi$, there exists a basis for $A$

$$
\mathcal{B}^{\prime}=\left\{c_{j i}^{\lambda} \mid \lambda \in \Lambda, i \in I(\lambda), j \in J(\lambda)\right\}
$$

defined by $\phi\left(a_{i j}^{\lambda}, c_{k l}^{\mu}\right)=\delta_{\lambda, \mu} \delta_{j k} \delta_{i l}$. A routine check shows that the following formulae hold (cf. (1.2.2)):

$$
\begin{cases}a \cdot c_{j i}^{\lambda} \equiv \sum_{j^{\prime} \in J(\lambda)} f_{\lambda, j}\left(j^{\prime}, a\right) c_{j^{\prime} i}^{\lambda} & \bmod \left(A_{\mathcal{B}^{\prime}}^{<\lambda}\right), \\ c_{j i}^{\lambda} \cdot a \equiv \sum_{i^{\prime} \in I(\lambda)} f_{i, \lambda}\left(a, i^{\prime}\right) c_{j i^{\prime}}^{\lambda} & \bmod \left(A_{\mathcal{B}^{\prime}}^{<\lambda}\right),\end{cases}
$$

where $A_{\mathcal{B}^{\prime}}^{<\lambda}$ is defined with respect to $\mathcal{B}^{\prime}$ (see the definition after (1.1.3)). Thus $\mathcal{B}^{\prime}$ is a standard basis for $A$ on $\Lambda$ with reversed partial order $\leqslant^{\prime}$, i.e., $x \leqslant \leqslant^{\prime} y$ if and only if $x \geqslant y$, and therefore $A$ is a standardly full-based algebra with respect to $\mathcal{B}^{\prime}$ by $(4.2 .1)$.

We now fix a compatible linear order $\left\{\lambda_{1}, \lambda_{2}, \ldots, \lambda_{m}\right\}$ for $\Lambda$ as in (1.1.2). Let $I_{i}=\operatorname{span}_{\mathrm{k}}\left\{\mathcal{B}^{\prime \lambda_{j}} \mid j \geqslant i\right\}$. Then

$$
0 \subset I_{m} \subset \cdots \subset I_{1}=A
$$

is a based filtration with respect to $\mathcal{B}^{\prime}$ and $\left(\Lambda, \leqslant^{\prime}\right)$, and hence, a heredity chain of $A$ by (3.2.2).

We claim that $\operatorname{hd}\left(I_{m}\right) \cong L\left(\lambda_{m}\right)^{\oplus r}$. Indeed, by taking $a=a_{k l}^{\mu}$ in (4.3.2) and noting (1.2.2), we have $f_{\lambda, j}\left(j^{\prime}, a\right)=0$ if $\mu \nless \lambda$. Thus, we have

$$
a_{k l}^{\mu} c_{j i}^{\lambda}=0 \bmod \left(A_{\mathcal{B}^{\prime}}^{\prime^{\prime} \lambda}\right) \text { for } \mu \nless \lambda .
$$

Suppose now that there exists a simple module $L(\lambda)$ in $\operatorname{hd}\left(I_{m}\right)$ such that $L(\lambda) \neq$ $L\left(\lambda_{m}\right)$; then $\lambda \nless \lambda_{m}$ as $\lambda_{m}$ is minimal. Since $A$ is quasi-hereditary, there exists $a \in A^{\lambda}$ such that $a \cdot L(\lambda) \neq 0$. However, (4.3.3) implies $a \cdot I_{m}=0$, a contradiction.

By the claim, $\lambda_{m}$ must be maximal in $\Lambda$. But it is also minimal. Thus, any maximal element in $\Lambda$ is also minimal. This implies that any two elements in $\Lambda$ are incomparable. Therefore, any standard module $\Delta(\lambda)$ in $A$ - $\bmod$ is simple and every PIM $P(\lambda)$ equals $\Delta(\lambda)$. Consequently, every PIM is simple and $A$ is semisimple. 
(4.3.4) Corollary. Let $A$ be the group algebra of a finite group $G$. If $A$ is split over $\mathrm{k}$ and char $(\mathrm{k})$ divides $|G|$, then $A$ is not quasi-hereditary.

Proof. This result follows from the fact that any group algebra is symmetric.

\section{Some standard Bases For $q$-Schur Algebras}

In this section, we shall construct two standard bases for $q$-Schur algebras as quasi-hereditary algebras. The first one is obtained by showing an existing basis to be a standard defining base. Thus, by (3.2.1), we obtain a new proof of the quasiheredity of $q$-Schur algebras. The second one is constructed inductively as in the proof of (4.2.3). We shall see that it agrees with the $q$-codeterminant basis. Thus, this in fact gives a new and much easier method to construct the $q$-codeterminant bases (cf. [Gr]).

$5.1 q$-Schur algebras. Let $\mathrm{k}$ be a commutative ring and $q \in \mathrm{k}$ invertible. A $q$ Schur algebra is defined similarly as the algebras $\mathcal{S}_{q}(W)$ described in (1.1.4(c)) for $W=\mathfrak{S}_{r}$, the symmetric group on $r$ letters. Let $\Lambda(n, r)$ be the set of all compositions of $r$ into $n$ parts (see (1.3.3)). For $\lambda, \mu \in \Lambda(n, r)$, the notation $W_{\lambda}, \mathcal{D}_{\lambda \mu}, \mathcal{D}_{\lambda \mu}^{+} \mathcal{D}_{\lambda}$, etc. has an obvious meaning as defined in (1.1.4(c)). Let $x_{\lambda}=\sum_{w \in W_{\lambda}} T_{w}$.

By definition, the $q$-Schur algebra $\mathcal{S}_{q}(n, r)$ of degree $(n, r)$ is the endomorphism algebra

$$
\mathcal{S}_{q}(n, r)=\mathcal{S}_{\mathrm{k}, q}(n, r)=\operatorname{End}_{\mathcal{H}}\left(\bigoplus_{\lambda \in \Lambda(n, r)} x_{\lambda} \mathcal{H}\right),
$$

where $\mathcal{H}=\mathcal{H}_{\mathrm{k}, q}$. So if $q=1$, then $\mathcal{S}_{1}(n, r)$ is the Schur algebra we introduced in (1.3.3).

The algebra $\mathcal{S}_{q}(n, r)$ has a natural basis like the $\xi$-basis for the Schur algebras:

$$
\left\{\phi_{\lambda \mu}^{w} \mid \lambda, \mu \in \Lambda(n, r), w \in \mathcal{D}_{\lambda \mu}\right\}
$$

where $\phi_{\lambda \mu}^{w}$ is defined by

$$
\phi_{\lambda \mu}^{w}\left(x_{\rho} h\right)= \begin{cases}\sum_{y \in W_{\lambda} w W_{\mu}} T_{y} h, & \text { if } \rho=\mu, \\ 0, & \text { otherwise }\end{cases}
$$

In [BLM] and [Du1] the Kazhdan-Lusztig basis for $\mathcal{S}_{\mathcal{A}, q}(n, r)$ is introduced for $\mathcal{A}:=\mathbb{Z}\left[t, t^{-1}\right]$ with $q=t^{2}$. Both bases are indexed by the set $\mathcal{D}(n, r)$ of all $W_{\lambda}-W_{\mu}$ double cosets for all $\lambda, \mu \in \Lambda(n, r)$. Note that we can easily identify $\mathcal{D}(n, r)$ with the set $\Omega$ defined in (1.3.3) and with the set $\left\{(\lambda, w, \mu) \mid \lambda, \mu \in \Lambda(n, r), w \in \mathcal{D}_{\lambda \mu}\right\}$.

Let us denote the Kazhdan-Lusztig basis by $\mathcal{B}=\left\{\theta_{D} \mid D \in \mathcal{D}(n, r)\right\}$. The definition of this basis is closely related to the $B_{w}$ basis (1.1.4(b)) for Hecke algebras (see [Du1]) over $\mathcal{A}$, and from [Du1, 3.4] we have

$$
\theta_{D} \theta_{D^{\prime}} \neq 0 \Rightarrow B_{w_{D}} B_{w_{D^{\prime}}} \neq 0
$$

where $w_{X}$ is the longest word of $X$. If we view $\mathrm{k}$ as an $\mathcal{A}$-module via $t^{2} \mapsto q$, then

$$
\mathcal{S}_{\mathrm{k}, q}(n, r)=\mathcal{S}_{\mathcal{A}, t^{2}}(n, r) \otimes_{\mathcal{A}} \mathrm{k} .
$$

The basis $\mathcal{B}$ gives a basis for $\mathcal{S}_{\mathrm{k}, q}(n, r)$ over an arbitrary k. Let us denote the induced bases for $\mathcal{H}_{\mathrm{k}, q}$ and $\mathcal{S}_{\mathrm{k}, q}(n, r)$ by the same notation. Clearly, (5.1.2) remains true. 
5.2 Robinson-Schensted correspondence. For $\lambda \in \Lambda^{+}(n, r)$, we may identify $\lambda$ with its corresponding diagram which consists of boxes arranged in a manner as illustrated by the example $\lambda=(421)$, for which we have

$$
\begin{gathered}
\lambda=\square \quad \square \quad \square \quad \square \\
\square \quad \square
\end{gathered}
$$

A $\lambda$-tableau $\mathbf{t}$ is obtained by replacing each box by one of the numbers $1,2, \cdots, r$, allowing no repeats. Let $\mathbf{t}^{\lambda}$ be the $\lambda$-tableau in which the numbers $1,2, \cdots, r$ appear in order along successive rows. The symmetric group $W=\mathfrak{S}_{r}$ acts on the set of $\lambda$-tableaux by letter permutations. For $i \in I(n, r)$ a $\lambda$-tableau obtained by putting the numbers $i_{1}, \cdots, i_{r}$ in the diagram of $\lambda$ in order along successive rows is called a $\lambda$-tableau of type $\mu=\operatorname{wt}(i)$. Let $T(\lambda, \mu)$ denote the set of all $\lambda$-tableaux of type $\mu$. A $\lambda$-tableau $\mathbf{t}$ is called semi-standard (resp. standard) if its entries are nondecreasing (resp. increasing) along each row and increasing along each column. We denote by $T_{0}(\lambda, \mu)$ the set of all semi-standard $\lambda$-tableaux of type $\mu$. Clearly, $T_{0}(\lambda, \omega)$ is the set of all standard $\lambda$-tableaux where $\omega=\left(1^{r}\right)$.

Let $w \mapsto(\mathbf{r}(w), \mathbf{s}(w))$ be the Robinson-Schensted map from $W$ to the set of pairs of standard tableaux (see, e.g., [B]). It is well-known (cf. [BV]) that the following holds: for $x, y \in W$ :

$$
x \sim_{L} y \quad\left(\text { resp. } x \sim_{R} y\right) \text { if and only if } \mathbf{s}(x)=\mathbf{s}(y) \quad(\text { resp. } \mathbf{r}(x)=\mathbf{r}(y)) .
$$

5.3 Kazhdan-Lusztig bases. From (1.1.4(b)) we see that $\mathcal{H}$ is a based algebra on the poset of two-sided cells of $W$. For symmetric groups, this poset is isomorphic to the poset of partitions $\lambda$ of $r$. For $w \in W$ and $h \in \mathcal{H}=\mathcal{H}_{\mathrm{k}, q}$, we have from [KL] that

$$
h B_{w} \in \sum_{z \leqslant L} \mathrm{k} B_{z} .
$$

Thus, if $w \in \lambda$ and we identify $w$ with $(\mathbf{r}, \mathbf{s})$ via the Robinson-Schensted correspondence, we have by (5.3.1) that

$$
h B_{(\mathbf{r}, \mathbf{s})} \equiv \sum_{\mathbf{u} \in T_{0}(\lambda, \omega)} \alpha_{\mathbf{u}}(h, \mathbf{r}) B_{(\mathbf{u}, \mathbf{s})} \quad \bmod \mathcal{H}^{>\lambda} .
$$

Using the involution $T_{w} \mapsto T_{w^{-1}}$, one sees easily that the basis $B_{w}(w \in W)$ satisfies (1.2.2). (The fact that $\alpha_{\mathbf{u}}(h, \mathbf{r})$ is independent of $\mathbf{s}$ can be seen as follows: For $\mathrm{k}=\mathcal{A}=\mathbb{Z}\left[t, t^{-1}\right]$ and a simple reflection $s$, we have by [KL, 4.2] that the coefficient $\alpha_{\mathbf{u}}\left(B_{s}, \mathbf{r}\right)$ defined by $w=(\mathbf{r}, \mathbf{s})$ is the same as defined by $w^{*}=\left(\mathbf{r}, \mathbf{s}^{\prime}\right)$ where the $*$ map is defined in $[\mathrm{KL}, 4.1]$. So the independence over $\mathcal{A}$, and hence over any $\mathrm{k}$, follows from the argument in $[\mathrm{KL}, \S 5]$.) So $\mathcal{H}$ in this case is a standardly based algebra. Here $I(\lambda)=J(\lambda)=T_{0}(\lambda, \omega)$.

We are now ready to prove that the KL bases $\mathcal{B}$ for a $q$-Schur algebra is a standard defining base.

(5.3.3) Theorem. The $q$-Schur algebra $\mathcal{S}_{q}(n, r)$ is a standardly based algebra on $\Lambda^{+}(n, r)$ with defining base $\mathcal{B}$. Moreover, $\mathcal{S}_{q}(n, r)$ is quasi-hereditary over $\mathrm{k}$.

Proof. By (1.1.4), $\mathcal{S}_{q}(n, r)$ is a based algebra on $\Lambda^{+}(n, r)$ with defining base $\mathcal{B}$.

For $\lambda, \mu \in \Lambda(n, r)$, let $\mathcal{D}_{\lambda \mu}^{+}$be the cross section of $W_{\lambda}-W_{\mu}$ double cosets of maximal length. Then, for $\mu \in \Lambda^{+}(n, r)$, if $\Gamma_{\mu}$ is the left cell of $W$ containing $w_{0, \mu}$, the longest element of $W_{\mu}$, then we may identify $\Gamma_{\mu}$ with the set $T_{0}(\mu, \omega)$ of standard 
$\mu$-tableaux, and $\mathcal{D}_{\lambda \mu}^{+} \cap \Gamma_{\mu}$ with the set $T_{0}(\mu, \lambda)$ of semi-standard $\mu$-tableaux of type $\lambda$ by [Du5, 3.2-3]. Let

$$
I(\mu)=J(\mu)=\bigcup_{\lambda} T_{0}(\mu, \lambda) .
$$

If $D=W_{\lambda} w W_{\nu} \in \mathcal{D}(n, r)$, then $w_{D} \in \mathcal{D}_{\lambda \nu}^{+}$. By identifying $\Lambda^{+}(n, r)$ with the set of the corresponding two-sided cells, we now assume $w_{D} \in \mu$. Then we have $\lambda \leqslant \mu$ and $\nu \leqslant \mu$. This follows from the relations $w_{0, \lambda} \geqslant_{R} w_{D}$ and $w_{0, \nu} \geqslant_{L} w_{D}$. Let $(\mathbf{r}, \mathbf{s})$ be the pair of standard $\mu$-tableaux corresponding to $w_{D}$; then both $\left(\mathbf{r}, \mathbf{t}^{\mu}\right)$ and $\left(\mathbf{t}^{\mu}, \mathbf{s}\right)$ define uniquely two elements $x^{-1}, y$ with $x, y \in \Gamma_{\mu}$ respectively. That is, $w_{0, \mu} \sim_{L} x^{-1} \sim_{R} w_{D}$ and $w_{0, \mu} \sim_{R} y \sim_{L} w_{D}$. In particular, we have $x^{-1} \in \mathcal{D}_{\nu \mu}^{+}$ and $y \in \mathcal{D}_{\lambda \mu}^{+}$. By $[\mathrm{Du} 5,3.3]$ there exist unique semi-standard tableaux $\mathbf{u} \in T_{0}(\mu, \nu)$ and $\mathbf{v} \in T_{0}(\mu, \lambda)$ defined by $x$ and $y$. In this way, we obtain a function

$$
f: \mathcal{D}(n, r) \rightarrow \bigcup_{\mu \in \Lambda^{+}(n, r)} I(\mu) \times I(\mu)
$$

sending $D$ to $f(D)=(\mathbf{u}, \mathbf{v})$ as described above. By $[D u 5,3.2-3]$ we see that $f$ is a bijection.

Now, if we let $\theta_{\mathbf{u}, \mathbf{v}}^{\mu}=\theta_{D}$, then one checks easily by (5.1.2) and (5.3.2) that $\mathcal{B}$ is a standard defining base for $\mathcal{S}_{q}(n, r)$.

If $\beta_{\lambda}$ is the corresponding bilinear form defined in (1.2.6), we have $\operatorname{im} \beta_{\lambda}=\mathrm{k}$ for all $\mathrm{k} \in \Lambda^{+}(n, r)$, since $\beta_{\lambda}\left(\theta_{W_{\lambda}}, \theta_{W_{\lambda}}\right)=1$. So the last assertion follows from (3.2.1).

5.4 Canonical and semi-standard bases for standard modules. The standard basis given in (5.3.3) for a $q$-Schur algebra gives rise to the so-called canonical bases for standard modules $\Delta(\mu)$, which are called traditionally $q$-Weyl modules. The following theorem was proved in [Du3]. A new and direct proof can be found in [Du6].

(5.4.1) Canonical Basis Theorem. For $\mu \in \Lambda^{+}(n, r)$, the set

$$
\left\{\theta_{D} z_{\mu} \mid D \in D(n, r)\right\} \backslash\{0\}
$$

forms a basis for the $q$-Weyl module $\Delta(\mu)$, where $z_{\mu} \in \Delta(\mu)$ is a vector of weight $\mu$.

So all the canonical bases form a local basis for standard modules (see (4.2.5)) and, by the approach described in (4.2.3), we can regard the Kazhdan-Lusztig basis in (5.3.3) is defined by the local canonical basis if we assume the quasi-heredity of $\mathcal{S}_{q}(n, r)$.

We now apply this idea to construct another standard basis for $\mathcal{S}_{q}(n, r)$ by the semi-standard bases of $q$-Weyl modules. Recall from [DJ1] the bijection $\partial$ : $T(\mu, \lambda) \rightarrow \mathcal{D}_{\lambda}$ defined by letting $\mathbf{t}^{\lambda} \partial(\mathbf{s})$ be the row-standard $\lambda$-tableau for which $i$ belongs to row $a$ if the place occupied by $i$ in $\mathbf{t}^{\mu}$ is occupied by $a$ in $\mathbf{s}$. The following result is due to Dipper and James [DJ1].

(5.4.2) Semi-standard Basis Theorem. For $\mu \in \Lambda^{+}(n, r)$, the $q$-Weyl module $\Delta(\mu)$ has a basis

$$
\left\{\phi_{\lambda \mu}^{\partial(\mathbf{s})} z_{\mu} \mid \lambda \in \Lambda(n, r), \mathbf{s} \in T_{0}(\mu, \lambda)\right\},
$$

where $z_{\mu} \in \Delta(\mu)$ is a vector of weight $\mu$. 
The relationship between this basis and the canonical basis is discussed in [Du5]. All semi-standard bases form a local basis for standard modules. We now use this local basis to construct another global standard basis for $\mathcal{S}_{q}(n, r)$.

$5.5 q$-Codeterminant bases - a new construction. Let $\phi_{\mu}=\phi_{\mu \mu}^{1}$. We order $\Lambda^{+}(n, r)$ in a manner as in (1.1.2) and let $e_{i}=\sum_{j \leqslant i} \phi_{\mu_{j}}$ and $J_{i}=\mathcal{S} e_{i} \mathcal{S}$, where $\mathcal{S}=\mathcal{S}_{q}(n, r)$. Then the sequence of ideals

$$
0=J_{0} \subseteq J_{1} \subseteq \cdots \subseteq J_{m}=\mathcal{S}
$$

is a heredity chain of the quasi-hereditary algebra $\mathcal{S}$ (see [CPS3, (3.7.2)]).

Let, for $\mathbf{u} \in T_{0}(\mu, \lambda)$ and $\mathbf{v} \in T_{0}(\mu, \nu)$,

$$
a_{\mathbf{u}, \mathbf{v}}^{\mu}=\phi_{\lambda \mu}^{\partial(\mathbf{u})} \phi_{\mu \nu}^{\partial(\mathbf{v})^{-1}} .
$$

(5.5.1) Theorem. The set $\left\{a_{\mathbf{u}, \mathbf{v}}^{\mu}\right\}$ forms a standard basis of $\mathcal{S}_{q}(n, r)$.

Proof. Let $\mathcal{S}_{i}=\mathcal{S} / J_{i-1}$. Then $J_{i} / J_{i-1}=\mathcal{S}_{i} \bar{e}_{i} \mathcal{S}_{i}$ is a heredity ideal of $\mathcal{S}_{i}$. Since $\mathcal{S}_{i} \bar{e}_{i}$ is isomorphic to $\Delta\left(\mu_{i}\right)$ and $\bar{e}_{i} \mathcal{S}_{i}$ to $\Delta^{\mathrm{op}}\left(\mu_{i}\right)$, where $\Delta^{\mathrm{op}}\left(\mu_{i}\right)$ is the standard right $\mathcal{S}$-module corresponding to $\mu_{i}$, by (5.4.2) we see that the set

$$
\left\{\phi_{\lambda \mu_{i}}^{\partial(\mathbf{s})} \bar{e}_{i} \mid \lambda \in \Lambda(n, r), \mathbf{s} \in T_{0}\left(\mu_{i}, \lambda\right)\right\}
$$

forms a basis for $\mathcal{S}_{i} \bar{e}_{i}$, and the similar result holds for $\bar{e}_{i} \mathcal{S}_{i}$. Therefore, the image in $\mathcal{S}_{i}$ of the set

$$
\left\{a_{\mathbf{u}, \mathbf{v}}^{\mu_{i}} \mid \mathbf{u} \in I\left(\mu_{i}\right), \mathbf{v} \in J\left(\mu_{i}\right)\right\}
$$

spans $J_{i} / J_{i-1}$, and therefore forms a basis by a comparison of ranks. Now, by induction, we obtain that $\left\{a_{\mathbf{u}, \mathbf{v}}^{\mu}\right\}$ is a basis for $\mathcal{S}$. Using the bimodule isomorphism

$$
\mathcal{S}_{i} \bar{e}_{i} \mathcal{S}_{i} \cong \Delta\left(\mu_{i}\right) \otimes_{\mathrm{k}} \Delta^{\mathrm{op}}\left(\mu_{i}\right)
$$

we can check easily that it is a standard defining base.

(5.5.2) Remark. The basis given in (5.5.1) is the same as the codeterminant basis of $\mathcal{S}_{q}(n, r)$ introduced in [Gr, §5] with a rather long and complicated proof. With the theory of standard based algebras, this basis is just a global basis determined by the local semi-standard basis for standard modules.

$5.6 q$-Borel subalgebras. The $q$-Borel subalgebras $\mathcal{S}_{q}^{\preccurlyeq}(n, r)$ and $\mathcal{S}_{q}^{\succcurlyeq}(n, r)$ of the $q$-Schur algebra $\mathcal{S}_{q}(n, r)$ have been introduced in [PW, 11.6] (compare (1.3.3)). By theorem (3.2.1), we can easily prove the following as in (1.3.3) by displaying a standard defining base.

(5.6.1) Theorem. The $q$-Borel subalgebras $\mathcal{S}_{q}^{\preccurlyeq}(n, r)$ and $\mathcal{S}_{q}^{\succcurlyeq}(n, r)$ (over $\left.\mathrm{k} !\right)$ are integrally quasi-hereditary.

\section{STANDARD DUALity AND INVOLUTIONS}

We will investigate standardly based algebras with dualities on their module categories, and will link this to possible cellular algebra structure on them. 
6.1 Duality. As in section one, $\mathrm{k}$ denotes a commutative ring with 1 . Let $A$ be a k-finite algebra. Let $\mathcal{C}$ be the category of left $A$-modules. If $\alpha$ is an antiautomorphism of $A$, then $\alpha$ induces a contravariant functor $D_{A, \alpha}$ on $\mathcal{C}$ defined as follows. For any $M \in \mathrm{Ob}(\mathcal{C})$, let

$$
M^{*}=\operatorname{Hom}_{\mathrm{k}}(M, \mathrm{k})
$$

be the linear dual of $M$. Then $M^{*}$ is a right $A$-module. Via $\alpha$, we may shift the right action to a left action. Thus we obtain a left $A$-module $D_{A, \alpha}(M)$. In other words, we have $D_{A, \alpha}(M)=M^{*}$ with $(a \phi)(m)=\phi(\alpha(a) m)$, for all $\phi \in M^{*}, a \in A$ and $m \in M$.

Recall that a duality on $\mathcal{C}=A$-mod for a finite dimensional algebra $A$ over a field $\mathrm{k}$ is a contravariant functor $D: \mathcal{C} \rightarrow \mathcal{C}$ such that if $D^{2} \cong i d_{\mathcal{C}}$, and $D$ is said to be a strong duality if $D$ is a duality such that $D(L) \cong L$ for any simple object $L \in \mathcal{C}$. This leads to the following definition.

(6.1.1) Definition. Let $A$ be a standardly based algebra over a commutative ring k. Let $\mathcal{C}$ be the category of k-finite (left) $A$-modules and let $\mathcal{C}_{f}$ be the full subcategory of $\mathrm{k}$-free objects. A contravariant functor $D: \mathcal{C} \rightarrow \mathcal{C}$ is said to be a standard duality if and

(a) the restriction of $D$ to $\mathcal{C}_{f}$ induces a functor $D: \mathcal{C}_{f} \rightarrow \mathcal{C}_{f}$ satisfying $D^{2} \cong i d_{\mathcal{C}_{f}}$,

(b) $D(\Delta(\lambda)) \cong \nabla(\lambda)$ for all $\lambda \in \Lambda$ (cf. (2.1.2)).

We have the following.

(6.1.2) Proposition. Let $\mathrm{k}$ be a field and let $A$ be a standardly full-based algebra over $\mathrm{k}$. If $D$ is a standard duality in the sense of (6.1.1), then $D$ is a strong duality.

Proof. Clearly, $D$ is a duality.

By the hypothesis and (3.2.1) we see that $A$ is a split quasi-hereditary algebra. So $\Delta(\lambda)$ and $\nabla(\lambda)$ defined in (2.1.2) agree with those defined in the corresponding highest weight category (see (3.2.2)). However, $D(\Delta(\lambda)) \cong \nabla(\lambda)$ is equivalent to $D(L(\lambda)) \cong L(\lambda)$. Therefore, $D$ is a strong duality.

Recall from (1.2.4) that a cellular algebra is a standardly based algebra with a certain anti-involution. This involution interchanges the left and right quasi-cells but leaves a two-sided quasi-cell unchanged. This motivates the following definition.

(6.1.3) Definition. Let $A$ be a standardly based algebra over a commutative ring k. An anti-involution $\alpha: A \rightarrow A$ is said to be standard if

(i) $D_{A, \alpha}$ is a standard duality on $A$-mod, and

(ii) for each $\lambda \in \Lambda$, there are $j_{0}, j_{1} \in J(\lambda)$ such that

$$
\mathrm{k} \mathcal{B}_{I(\lambda), j_{0}} \alpha\left(\mathrm{k} \mathcal{B}_{I(\lambda), j_{1}}\right) \equiv \mathrm{k} \mathcal{B}^{\lambda} \quad \bmod \left(A^{>\lambda}\right),
$$

where $\mathrm{k} \mathcal{B}_{*, *}$ denotes the free $\mathrm{k}$-submodule of $A$ spanned by $\mathcal{B}_{*, *}$. We shall call a pair $\left(j_{0}, j_{1}\right)$ with the above property a $\lambda$-pair.

We shall see that the condition (ii) guarantees that $\alpha$ interchanges the left and right quasi-cells defined by a certain standard defining base.

(6.1.4) Proposition. Let $\mathrm{k}$ be a commutative ring with 1 . Let $A$ be a standardly based algebra on the poset $\Lambda$ with defining base $\mathcal{B}=\left\{a_{i j}^{\lambda}\right\}$. If $A$ has a standard involution $\alpha$, then the set

$$
\mathcal{B}_{\alpha}=\left\{a_{i j_{0}}^{\lambda} \alpha\left(a_{j j_{1}}^{\lambda}\right) \mid \lambda \in \Lambda, i, j \in I(\lambda)\right\},
$$


where $\left(j_{0}, j_{1}\right)$ 's are a fixed $\lambda$-pair for each $\lambda$, is a standard defining base for $A$, and $\mathcal{B}_{\alpha}$ and $\alpha\left(\mathcal{B}_{\alpha}\right)$ are equivalent. Moreover, if the $\lambda$-pair $\left(j_{0}, j_{1}\right)$ can be chosen satisfying $j_{0}=j_{1}$ for each $\lambda \in \Lambda$, then $A$ is a cellular algebra.

Proof. By the definition, it is easy to see that the image of

$$
\mathcal{B}_{\alpha}^{\lambda}=\left\{a_{i j_{0}}^{\lambda} \alpha\left(a_{j j_{1}}^{\lambda}\right) \mid i, j \in I(\lambda)\right\}
$$

in $A / A^{>\lambda}$ is a spanning set of $A^{\lambda}$, and hence a basis since $\# \mathcal{B}_{\alpha}^{\lambda}=\operatorname{rank}_{\mathrm{k}} A^{\lambda}$ by (6.1.1)(b). Consequently, the set $\mathcal{B}_{\alpha}$ is a k-basis for $A$. It is also clear that $\mathcal{B}_{\alpha}$ is a standard defining base of $A$.

Since both $\mathcal{B}_{\alpha}$ and $\alpha\left(\mathcal{B}_{\alpha}\right)$ determine the same local basis for standard modules (cf. (2.1.4)), they are equivalent by (4.2.6).

The last assertion is obvious.

6.2 A special case. We now look at the special case where $A$ is standardly fullbased and the involution interchanges left and right quasi-cell modules.

(6.2.1) Proposition. Let $\mathrm{k}$ be a commutative local noetherian ring and let $A$ be a standardly full-based algebra over $\mathrm{k}$. If $\alpha: A \rightarrow A$ is an anti-involution satisfying $\alpha\left(\mathrm{k} \mathcal{B}_{I(\lambda), j}\right)=\mathrm{k} \mathcal{B}_{i(j), J(\lambda)}$ for all $\lambda \in \Lambda$ and $j \in J(\lambda)$, then $\alpha$ is a standard involution. Moreover, if, for each $\lambda \in \Lambda$, there are $j, j_{1} \in J(\lambda)$ such that $f\left(j, i\left(j_{1}\right)\right)$ is a unit in $\mathrm{k}$, then $A$ is cellular. (We shall call such an involution a distinguished standard involution.)

Proof. We first note that the condition on $\alpha$ induces a bijection from $J(\lambda)$ to $I(\lambda)$ sending $j$ to $i(j)$. So we may assume $I(\lambda)=J(\lambda)$ and $\alpha\left(\mathrm{k} \mathcal{B}_{I(\lambda), j}\right)=\mathrm{k}_{j, I(\lambda)}$ for all $j \in J(\lambda)$.

Since $\mathrm{k}$ is local and $A$ is standardly full-based, we may find a pair $\left(i_{0}, j_{0}\right) \in$ $I(\lambda) \times I(\lambda)$ for every $\lambda$ such that $f_{\lambda}\left(j_{0}, i_{0}\right)$ is a unit in $\mathrm{k}$. Thus we obviously have

$$
\mathrm{k} \mathcal{B}_{I(\lambda), j_{0}} \alpha\left(\mathrm{k} \mathcal{B}_{I(\lambda), i_{0}}\right) \equiv \mathrm{k}^{\lambda} \quad \bmod \left(A^{>\lambda}\right) .
$$

We now prove that $D_{A, \alpha}$ is a standard duality on $A$-mod. For any $M \in \mathrm{Ob} \mathcal{C}_{f}$, the freeness of $M$ implies $M^{* *} \cong M$ as k-modules. Since $\alpha^{2}=1$, we have a left $A$-module isomorphism $D_{A, \alpha}^{2} M \cong M$ by the definition of $D_{A, \alpha}$. This shows that $D_{A, \alpha}^{2} \cong i d_{\mathcal{C}_{f}}$. On the other hand, the hypothesis implies a k-module isomorphism $\alpha_{\lambda}: \Delta(\lambda) \rightarrow \Delta^{\mathrm{op}}(\lambda)$ satisfying $\alpha_{\lambda}(v) a=\alpha_{\lambda}(\alpha(a) v)$ for all $a \in A$ and $v \in \Delta(\lambda)$, and hence a $\mathrm{k}$-isomorphism

$$
\left.\operatorname{Hom}_{\mathrm{k}}(\Delta(\lambda), \mathrm{k})\right) \cong \operatorname{Hom}_{\mathrm{k}}\left(\Delta^{\mathrm{op}}(\lambda), \mathrm{k}\right) .
$$

Let $\left\{g_{j}^{\lambda}\right\}$ and $\left\{h_{j}^{\lambda}\right\}$ be the dual bases of $\left\{a_{j}^{\lambda}\right\}$ and $\left\{\alpha_{\lambda}\left(a_{j}^{\lambda}\right)\right\}$ respectively. Let $f$ : $g_{i}^{\lambda} \mapsto h_{i}^{\lambda}$, and extend it linearly to the Hom spaces. Then, by (2.1.3), we have

$$
a g_{i}^{\lambda}\left(a_{j}^{\lambda}\right)=g_{i}\left(\alpha(a) a_{j}^{\lambda}\right)=f_{i, \lambda}(\alpha(a), j)
$$

and

$$
a h_{i}^{\lambda}\left(\alpha_{\lambda}\left(a_{j}^{\lambda}\right)\right)=h_{i}^{\lambda}\left(\alpha_{\lambda}\left(a_{j}^{\lambda}\right) a\right)=h_{i}^{\lambda}\left(\alpha_{\lambda}\left(\alpha(a) a_{j}^{\lambda}\right)\right)=f_{i, \lambda}(\alpha(a), j)
$$

for any $a_{j}^{\lambda} \in \Delta(\lambda)$. Therefore, we have $a f\left(g_{i}^{\lambda}\right)=f\left(a g_{i}^{\lambda}\right)$. This shows that $f$ is an isomorphism of $A$-modules, and consequently, $D_{A, \alpha}(\Delta(\lambda)) \cong \nabla(\lambda)$ for all $\lambda \in \Lambda$. This proves that $D_{A, \alpha}$ is a standard duality, and hence $\alpha$ is a standard involution.

The last assertion follows immediately. 
(6.2.2) Corollary. Let $A$ be a split quasi-hereditary algebra over a commutative local noetherian ring. Then $A$ is a cellular algebra if and only if $A$ has a distinguished standard involution.

Proof. Clearly, if $A$ is cellular, then the associated involution is a distinguished standard involution.

\section{REFERENCES}

[B] C. Berge, Principles of Combinatorics, Academic Press, New York 1971. MR 42:5805

[BV] D. Barbasch and D. Vogan, Primitive ideals and orbital integrals in complex classical groups, Math. Ann. 259 (1982), 153-199. MR 83m:22026

[BLM] A.A. Beilinson, G.Lusztig, and R. Macpherson, A geometric setting for the quantum deformation of $G L_{n}$, Duke Math. J. (1990), 655-677. MR 91m:17012

[CPS1] E. Cline, B. Parshall and L. Scott, Finite dimensional algebras and highest weight categories, J. Reine Angew. Math. 391 (1988), 85-99. MR 90d:18005

[CPS2] E. Cline, B. Parshall and L. Scott, Duality in highest weight categories, Contemp. Math. 82 (1989), 7-22. MR 90g:17014

[CPS3] E. Cline, B. Parshall and L. Scott, Integral and graded quasi-hereditary algebras, I, J. Algebra. 131 (1990), 126-160. MR 91c:16009

[DJ1] R. Dipper and G. James, Representations of Hecke algebras of general linear groups, Proc. London Math. Soc. 52 (1986), 20-52. MR 88b:20065

[DJ2] R. Dipper and G. James, The q-Schur algebra, Proc. London Math. Soc. 59 (1989), 23-50. MR 90g:16026

[DJ3] R. Dipper and G. James, $q$-tensor spaces and $q$-Weyl modules, Trans. Amer. Math. Soc. 727 (1991), 251-282. MR 91m:20061

[DR1] V. Dlab and C. M. Ringel, Quasi-hereditary algebras, Illinois J. Math. 33 (1989), 280-291. MR 90e: 16023

[DR2] V. Dlab and C. M. Ringel, The module theoretical approach to quasi-hereditary algebras, Representations of Algebras and Related Topics (H. Tachikawa and S. Brenner, eds.) London Math. Soc. Lecture Note Ser., vol. 168, Cambridge Univ. Press, Cambridge, 1992, pp. 200-224. MR 94f: 16026

[Du1] J. Du, Kazhdan-Lusztig bases and isomorphism theorems for q-Schur algebras, Contemp. Math. 139 (1991), 121-140. MR 94b:17019

[Du2] J. Du, Integral Schur algebras for $G L_{2}$, Manuscripta Math. 75 (1992), 411-427. MR 93g:20086

[Du3] J. Du, Canonical basis for irreducible representations of quantum $G L_{n}$, Bull. London Math. Soc. 24 (1992), 325-334. MR 93g:17023

[Du4] J. Du, IC bases and quantum linear groups, Proc. Sympo. Pure.Math. 56 (1994), part 2, 135-148. MR 95d:17010

[Du5] J. Du, Canonical basis for irreducible representations of quantum $G L_{n}$, II, J. London Math. Soc. (2) 51 (1995), 461-470. MR 96h:17016

[Du6] J. Du, A new proof for the canonical bases of type A, preprint, UNSW.

[DS] J. Du and L. Scott, Lusztig conjectures, old and new, I, J. Reine Angew. Math. 455 (1994), 141-182. MR 95i:20062

[G1] J. A. Green, Polynomial Representations of $G L_{n}$, Lecture Notes in Math. 830, SpringerVerlag, Berlin, 1980. MR 83j:20003

[G2] J. A. Green, On certain subalgebras of the Schur algebras, J. Algebra 131 (1990), 265-280. MR 91b:20019

[G3] J. A. Green, Combinatorics and the Schur algebra, J. Pure Appl. Algebra 88 (1993), 89-106. MR 94g:05100

[Gr] R. Green, q-Schur algebras and quantized enveloping algebras, Thesis (1994).

[GL] J. Graham and G. Lehrer, Cellular algebras, Invent. Math. 123 (1996), 1-34. MR 97h:20016

[K] S. König, A criterion for quasi-hereditary, and an abstract straightening formula, Invent. Math. 127 (1997), 481-488. MR 97m:16019

[KL] D. Kazhdan and G. Lusztig, Representation of Coxeter groups and Hecke algebras, Invent. Math. 53 (1979), 155-174. MR 81j:20006 
[Lu] G. Lusztig, Introduction to quantum groups, Birkhäuser, Boston, 1993. MR 94m:17016

[PW] B. Parshall and J. Wang, Quantum linear groups, Memoirs Amer. Math. Soc. 89 (1991), No. 439. MR 91g: 16028

[S] L. Scott, Simulating algebraic geometry with algebra, I, Proc. Sympos. Pure Math. 47 (1987), part 2, 271-281. MR 89c:20062a

Schools of Mathematics, University of New South Wales, Sydney, 2052, Australia

E-mail address: jied@maths.unsw.edu.au

Department of Mathematics, University of Shanghai for Science \& Technology, Shanghai, 200093, People's Republic of China

E-mail address: hbruik@online.sh.cn 